\title{
Strategic Formation of Homogeneous Bargaining Networks ${ }^{\text {th }}$
}

\author{
F. Gauer ${ }^{\mathrm{a}, *}$, T. Hellmann ${ }^{\mathrm{a}}$ \\ ${ }^{a}$ Center for Mathematical Economics (IMW), Bielefeld University, P.O. Box 100131, D-33501 Bielefeld
}

\begin{abstract}
We study a model of strategic network formation prior to a Manea (2011a) bargaining game: ex-ante homogeneous players form costly undirected links, anticipating expected equilibrium payoffs from the subsequent bargaining game. Assuming patient players, we provide a complete characterization of generically pairwise stable networks: specific unions of separated pairs, odd circles, and isolated players constitute this class. We also show that many other structures, such as larger trees or unbalanced bipartite networks, cannot be pairwise stable at all. The analysis implies that the diversity of possible bargaining outcomes is small in (generically) pairwise stable networks.
\end{abstract}

Keywords: Bargaining, Network Formation, Noncooperative Games JEL-Classification: C72, C78, D85

\section{Introduction}

Markets often do not satisfy the assumptions imposed by general equilibrium theory. For instance, not all buyers may be able to trade freely with all sellers. Instead, the opportunity to create a joint surplus between two participants in a market may be influenced by several frictions such as the availability of information about trade opportunities, transportation costs, heterogeneous preferences, etc. Recent literature has paid particular attention to markets where opportunities to create a joint surplus are restricted to a network (see e.g., Manea, 2016, for a comprehensive overview of this literature). For such markets, it is shown that the law of one price cannot be upheld. Rational choice of market participants, instead, gives rise to bargaining over the division of the surplus created by the bilateral interactions, implying that prices can be heterogeneous and depend on the location of the trade in the network.

The literature has focused almost exclusively on settings where the network, representing bargaining opportunities, is exogenously given. ${ }^{1}$ However, if agents receive payoffs from bargaining such that for the outcome of the bargaining game agents' network positions matter (see e.g. Manea, 2011a, where equilibrium payoffs only depend on the network position and

\footnotetext{
${ }^{*}$ Corresponding author

Email addresses: fgauer86@gmail.com (F. Gauer), tim.hellmann@uni-bielefeld.de (T. Hellmann)

URL: https://sites.google.com/site/thellmannimw/ (T. Hellmann)

${ }^{1}$ See Section 1.1 for literature that also models network formation.
} 
the discount factor), then agents will have incentives to influence the network structure. In such situations, the underlying network should not be regarded as being exogenously given but as the outcome of strategic interaction among agents. We fill this gap in the literature by allowing agents to create the network prior to market interaction. This will be particularly relevant in markets where potential gains from trade are large relative to other exogenous reasons for network creation. However, such a market will not be without frictions, as interactions can be costly which may be motivated by time and effort required to initiate a relationship.

In such a setting, agents face a trade-off when forming links in the network: while new connections give rise to additional outside options via new trade opportunities, agents also incur the cost of network formation. Which networks can we expect to form depending on the costs of links relative to joint surplus? The answer to this question has fundamental implications for the induced bargaining outcome. Can we expect to observe heterogeneous bargaining outcomes implied by heterogeneous network positions? Or will ex-ante homogeneous players rather form networks that ensure equitable payoffs?

To answer these questions, we set up a sequential model of strategic network formation prior to a Manea (2011a) infinite-horizon network bargaining game. Ex ante homogeneous players first form undirected, costly links. In the second stage, the resulting network is taken as given and players sequentially bargain with neighbors for the division of a mutually generated unit surplus. According to Manea (2011a), all subgame perfect equilibria of the bargaining game are payoff equivalent. To abstract from issues of time preferences, we additionally assume that players are infinitely patient in the bargaining stage. The resulting limit equilibrium payoff in the bargaining stage can be obtained employing an algorithm that has been developed in Manea (2011a) and which only depends on the network position of each player. Anticipating equilibrium outcomes in the second stage, players then interact strategically in the network formation stage.

We use the seminal approach of Manea (2011a) to model market interaction for several reasons. First, Manea does not restrict the set of networks to bipartite networks (i.e. buyer seller networks) and does not characterize equilibrium outcomes for only a restricted set of networks. In particular, none of the distorting effects are present that might otherwise arise from additional incentives to add or delete links, e.g. from ex-ante heterogeneity among players, or in buyer-seller scenarios. Second, Manea's network bargaining game is analytically tractable and has some well behaved equilibrium properties. Subgame perfect equilibria are payoff equivalent for any level of time discount, implying for our setting a unique equilibrium payoff in the second stage for any network formed in the first stage. When players become patient, Manea characterizes limit equilibrium payoffs by developing an equally convenient and sophisticated algorithm. We make extensive use of this algorithm and contribute to a deeper understanding of its features throughout this paper. Finally, because of the stationarity assumption that we share in our approach, stochastic effects do not play a role for equilibrium payoff. Instead, the limit equilibrium payoff of the bargaining stage solely de- 
pends on the network position, allowing to model network formation prior to the bargaining stage in a well defined sense.

To characterize resulting network structures, we use the notion of pairwise stability introduced in Jackson and Wolinsky (1996). We further distinguish between network structures that are pairwise stable for a range of cost levels, denoted as generically pairwise stable, and networks that are only stable for a single cost level, called nongenerically pairwise stable. We present necessary and sufficient conditions for networks to be pairwise stable (Theorems 1, 2 and 3) and are able to deduce a complete characterization of the set of generically pairwise stable networks as our first main result (Corollary 3 ). The empty network is uniquely pairwise stable for high costs of network formation. For intermediate cost ranges a network is generically pairwise stable if and only if it consists of connected pairs of players, sometimes also called a matching, (and an isolated player, if the number of players is odd). For smaller costs of network formation, also larger components than just pairs may occur such that a network is generically pairwise stable if and only if any nonsingleton component is either an odd circle (where the number of admissible players in the component is inversely related to the cost of network formation), or a connected pair, and there exists at most one isolated player. This result also establishes the existence of pairwise stable networks at each level of linking costs. We observe only relatively sparse network structures since disagreement links cannot be contained in a pairwise stable network (Proposition 1). A disagreement link denotes a link which is never used to generate surplus in the bargaining stage and therefore does not yield any value while causing costs.

Nongenerically pairwise stable networks only occur once at a given cost level since players are exactly indifferent between forming a link or not. Therefore, richer structures may be nongenerically pairwise stable. However, we are able to substantially narrow down this set of networks by revealing that no component of such a network can be too unbalanced or too intraconnected, can be a tree (with more than three players), or can contain a particular "cut-player" whose removal results in a larger number of components (Proposition 2).

Beyond the insights into the structure of pairwise stable networks, we study the implications for the bargaining outcomes. As our second main result, we conclude from our complete characterization that pairwise stability substantially narrows down the diversity of induced bargaining outcomes among players (Corollary 4). More specifically, all players with at least one link in generically pairwise stable networks receive the same limit equilibrium payoff in the bargaining stage. In that sense, networks - when restricted to the set of connected players - are equitable. However, in generically pairwise stable networks, players may very well incur different network formation costs to obtain the same limit equilibrium payoff in the bargaining stage. One possible left-over isolated player may also exist, receiving a limit equilibrium payoff of 0 in the bargaining stage. Nongenerically pairwise stable networks, however, admit heterogeneous limit equilibrium payoffs in the bargaining stage, although players are ex ante homogeneous. 


\subsection{Related Literature}

Besides Manea (2011a), a substantial literature developed recently that studies models of market interaction on networks. Particular attention has been paid to the bargaining process determining prices.

The study of bargaining problems has a long tradition in the economic literature and dates back to the work of Nash $(1950,1953)$. Modeling bargaining more explicitly, Rubinstein (1982) introduces the well known extensive game between two players with alternating offers. Extending this approach to a setting of two populations (e.g. buyers and sellers) with constant sizes, Rubinstein and Wolinsky (1985) show that outcomes of the bargaining process depend on outside options such as matching probabilities. The assumption that markets are stationary in the sense that population sizes stay constant over time is critical for their result as Gale (1987) shows. The work of Manea (2011a), to which we add a preceding stage of strategic interaction, can, hence, be regarded as an extension of these seminal papers. Assuming stationarity, bargaining power is endogenized in a natural and well-defined manner as an outcome of the given network structure and the respective player's position in it.

There are other approaches to model bargaining on networks and the outcomes depend on the assumed bargaining protocol. In the early work of Corominas-Bosch (2004) on bargaining on networks, buyers and sellers make public offers although trade is restricted to the network. It is shown that trade is efficient in the sense that the maximal number of trades occur and that payoffs depend on the network position. This result extends to a one-population model with a centralized matching procedure that selects trading links from the network efficiently, as Polanski (2007) shows. Allowing for decentralized matching, Abreu and Manea (2012a) and Abreu and Manea (2012b) study a model similar to Manea (2011a), but without relying on network stationarity. Instead, players who reach an agreement are not replaced. In such a model, agreements are not necessarily reached immediately in equilibrium nor are agreements efficient. Because of the additional complexity that arises due to stochastic effects once the stationarity assumption is relaxed, it is not possible to derive a closed form solution of expected payoffs and, hence, there is little hope to study network formation prior to such a bargaining model. Instead, the assumption of network stationarity ensures a tractable solution of the bargaining stage in Manea (2011a).

Studying network formation when incentives are determined by the bargaining outcome, we also contribute to the literature on strategic network formation which has been inspired by the seminal paper of Jackson and Wolinsky (1996). Other prominent works which have been carried out since then (although not in a bargaining framework) are those by Bala and Goyal (2000), Calvó-Armengol (2004), Galeotti et al. (2006), Goyal and Joshi (2003, 2006), and Watts (2001), to name a few. Some effort has also been dedicated to gaining rather general insights regarding the existence, uniqueness, and structure of stable networks; see, for example, Hellmann (2013) and Hellmann and Landwehr (2014). ${ }^{2}$

\footnotetext{
${ }^{2}$ Note, however, that the results of Hellmann (2013) and Hellmann and Landwehr (2014) are in general
} 
There is only little literature studying market interaction in endogenous networks. In one early approach by Kranton and Minehart (2001), buyers can form costly links to sellers, prior to a draw of private values and an ascending price auction. Pairwise stable networks are shown to maximize expected welfare. However, this result is not robust to bilateral link formation or split of costs between buyers and sellers, as Elliott (2015) shows. Market interaction in terms of auctions also yields fundamentally different results than bargaining as all the gains from trade are obtained by one side of the market. In endogenous buyerseller networks where outcomes are the result of bargaining instead of auctions, Polanski and Vega-Redondo (2013) show inefficiency of stable networks. Both the payoff resulting from bargaining and the restriction to a buyer-seller setup in these papers differ from our model.

There is also some literature studying network formation prior to a bargaining game without restricting to the bipartite nature of buyer-seller networks. Calvó-Armengol (2003) considers a similar framework as ours. In the first stage, players form the network, on which they bargain in the second stage. The bargaining protocol, however, differs from Manea (2011a) in several crucial aspects since bargaining ends after one agreement has been formed and a selection function is chosen randomly to determine the sequence of offers. With a given selection function, the equilibria in the bargaining stage are extremely simple depending only on the first mover advantage. As a consequence, a player's network position affects bargaining power only in terms of the probability of being selected as a proposer or responder. This leads to a characterization of pairwise stable networks in which the players' neighborhood size is the only relevant feature of the network structure. In our approach, instead, the network influences payoffs in a more complex way resulting from the Manea (2011a) bargaining game. The results in Calvó-Armengol (2003) therefore differ substantially from our results though we both share the assumption of costly links.

Trade between possibly distant but connected players in an endogenous network is modeled in Condorelli and Galeotti (2012). Since the length of the trading path does not affect the surplus, only minimal networks form and results can be compared to the two way flow model in Bala and Goyal (2000). In our model, instead, the opportunity to create joint surplus only arises between directly connected players.

Most closely related to our approach, may be an extension in the online appendix of Manea (2011a), referred to as Manea (2011b). There, Manea also studies network formation prior to his bargaining game in the second stage, but assumes no cost of link formation. A network is then pairwise stable if and only if it is equitable meaning that all players receive the same payoff. We complement this approach by asking whether we can expect the same networks to form even if links are costly. ${ }^{3}$

not applicable to our framework, because our model does not include certain crucial conditions that would permit such an application. For details, see Gauer (2016, Appendix 3.B).

${ }^{3}$ In fact, we show that only "skeletons" of equitable networks (that is, certain unions of separated pairs and odd circles) survive if costs are positive. However, nonequitable networks, such as unions of odd circles and an isolated player, can also be pairwise stable in our setting. 
For a more comprehensive survey of the literature on market interaction on networks, the reader may be referred to Manea (2016).

The rest of the paper is organized as follows. In Section 2 we introduce the model, including the decisive results of Manea (2011a). The main results on the structure of stable networks and induced bargaining outcomes are developed in Section 3. Finally, Section 4 concludes. The more evolved proofs are presented in Appendix A.

\section{The Model}

Consider a finite set of ex ante identical players $N=\{1,2, \ldots, n\}, n \geq 3$, and discrete time periods $t=0,1,2, \ldots$ of interaction. In the initial period $t=0$, players form the network and in periods $t=1,2, \ldots$ engage in an infinite horizon bargaining game over the division of a unit surplus generated by the links.

We denote a connection or link between two players $i, j \in N, i \neq j$ by $\{i, j\}$ which we abbreviate for simplicity by $i j=j i:=\{i, j\}$. We then define the complete network $g^{N}=\{i j \mid i, j \in N, i \neq j\}$ as the network where any two players are connected to each other and the set of all undirected networks by $G=\left\{g \mid g \subseteq g^{N}\right\}$. Let $N_{i}(g):=\{j \in N \mid i j \in g\}$ denote the set of player $i$ 's neighbors in $g$ and let $\eta_{i}(g):=\left|N_{i}(g)\right|$ be its cardinality which is also referred to as the degree of player $i$. Furthermore, for a network $g$, a set $C \subseteq N$ is said to be a component if there exists a path between any two players in $C$ and it is $N_{j}(g) \cap C=\emptyset$ for all $j \notin C{ }^{4}$ The set of all components of $g$ then defines a partition of the player set $N$. A subnetwork $g^{\prime} \subseteq g$ is said to be component-induced if there exists a component $C$ of $g$ such that $g^{\prime}=\left.g\right|_{C}$ where the network $\left.g\right|_{K}:=\{i j \in g \mid i, j \in K\}$ is the subnetwork restricted to the player set $K \subset N$. In addition, for two networks $g, g^{\prime} \subseteq g^{N}$ let $g-g^{\prime}:=g \backslash g^{\prime}\left(g+g^{\prime}:=g \cup g^{\prime}\right.$, respectively) denote the network obtained by deleting the set of links $g^{\prime} \cap g$ from (adding the set of links $g^{\prime} \backslash g$ to) the network $g$.

The bargaining stage taken from Manea (2011a) can be described as follows. After the network has been formed in period $t=0$, nature randomly chooses one link $i j \in g$ in each period $t=1,2, \ldots$ with equal probability. ${ }^{5}$ The chosen link generates the surplus that both involved players can divide among themselves. One of the two players is randomly assigned the role of the proposer while the other one is selected as responder, again with equal probability. The proposer makes an offer how to distribute the unit surplus while the responder can accept or reject the offer: If the offer is rejected, then both players receive a

\footnotetext{
${ }^{4}$ We say that there exists a path between two players $i^{\prime}, i^{\prime \prime} \in N$ in $g$ if there exist players $i_{1}, i_{2}, \ldots, i_{\bar{m}} \in N$, $\bar{m} \in \mathbb{N}$, such that $i_{1}=i^{\prime}, i_{\bar{m}}=i^{\prime \prime}$ and $i_{m} i_{m+1} \in g$ for $m=1,2, \ldots, \bar{m}-1$.

${ }^{5}$ Manea (2011a) allows for an arbitrary link selection probability distribution (with full support) in the bargaining stage. However, to calculate payoffs in examples and to model network formation (see Manea, 2011b), Manea often relies on the "uniform matching technology" that selects links with equal probability. For ease of notation, we also rely on this uniform matching technology. This is done w.l.o.g. since the probability distribution over links does not affect payoffs in the limit $\delta \rightarrow 1$ as long as it has full support. Further, we save notation for not having to define a new link selection probability distribution for every possible network, giving rise to a simple calculation of equilibrium payoffs, see (1).
} 
payoff of zero and stay in the game whereas both leave with the agreed shares if the offer is accepted. In the latter case, both players get replaced one-to-one in the next period such that the initially formed network remains unchanged. ${ }^{6,7}$ The network structure, as well as all offers and responses are common knowledge. A player's strategy in this extensive form game pins down the offer she makes as proposer and the answer she gives as responder after each possible history of the game. Based on this, a player's payoff is then given by her discounted expected agreement share. A strategy profile is said to be a "subgame perfect equilibrium" of the bargaining game if it induces Nash equilibria in subgames following every history. Players are assumed to discount time by a uniform discount factor $\delta \in(0,1)$.

A key result in Manea is that all subgame perfect equilibria are payoff equivalent and that each player's equilibrium payoff exclusively depends on her network position and the discount factor $\delta$ (see Manea, 2011a, Theorem 1). Moreover, the equilibrium payoff vector, which we denote as $v^{* \delta}(g)=\left(v_{i}^{* \delta}(g)\right)_{i \in N}$, is the unique solution to the equation system

$$
v_{i}=\left(1-\frac{\eta_{i}(g)}{2 d^{\#}(g)}\right) \delta v_{i}+\sum_{j \in N_{i}(g)} \frac{1}{2 d^{\#}(g)} \max \left\{1-\delta v_{j}, \delta v_{i}\right\}, \quad \forall i \in N,
$$

where $d^{\#}(g)$ denotes the total number of links in the network $g$. To see why equilibrium payoff has to satisfy (1), note that by the stationarity assumption there exists a subgame perfect equilibrium where strategies are such that each player $i$ always accepts any offer that gives her at least her continuation value $\delta v_{i}$ and always makes the same proposal (depending on the identity of responder). In the latter case, she has the first mover advantage and can extract all the remaining surplus from the match. Thus, she offers to $j$ his continuation value $\delta v_{j}$ if her own resulting share, $1-\delta v_{j}$, is not below her continuation value $\delta v_{i}$. The probability with which $i$ is selected to propose to $j$ is given by $\frac{1}{2 d^{\#}(g)}$. Hence, equilibrium payoffs have to satisfy (1) and are in fact the unique solution to this system of equations. This implies that an agreement between $i$ and $j$ is only feasible in equilibrium if $\delta\left(v_{i}^{* \delta}(g)+v_{j}^{* \delta}(g)\right) \leq 1$ giving rise to the definition of the equilibrium agreement network $g^{* \delta}:=\left\{i j \in g \mid \delta\left(v_{i}^{* \delta}(g)+v_{j}^{* \delta}(g)\right) \leq 1\right\}$.

Throughout this paper, we abstract from issues of time preferences in order to have equilibrium payoffs depend solely on the network structure. Thus, we focus on the limit case of $\delta \rightarrow 1$, meaning that players are infinitely patient. For $\delta$ large enough, Manea (2011a, Theorem 2) finds that the equilibrium agreement network $g^{* \delta}$ does not change in response to a change in $\delta$ taking the network $g$ as given, i.e. after the network $g$ has been formed in the first stage. This network $g^{*} \subseteq g$ is then called the limit equilibrium agreement network. Moreover, Manea (2011a, Theorem 2) also implies that the limit equilibrium payoff vector

\footnotetext{
${ }^{6}$ The replacement assumption is primarily due to technical reasons. The implication that the network structure does not change over time makes the model analytically tractable. See also the discussion on models without replacement, e.g. Abreu and Manea (2012b), in Section 1.1.

${ }^{7}$ Because of replacement after successful agreements, Manea carefully distinguishes between network positions and (potentially) different players being in one and the same position in different periods. However, as we examine solely the stage of network formation at time $t=0$ here, we can neglect this distinction.
} 
$v^{*}(g):=\lim _{\delta \rightarrow 1} v^{* \delta}(g)$ is well-defined, i.e. it always exists.

Manea develops a smart algorithm to determine the limit equilibrium payoff vector $v^{*}(g)$ and we make heavily use of this computational method. To prepare for the implementation of the algorithm we need to introduce some additional notation. For any set of players $M \subseteq N$ and any network $g$ let $L^{g}(M):=\{j \in N \mid i j \in g, i \in M\}$ be the corresponding partner set in $g$, that is the set of players having a link to a player in $M$. Further, a set $M \subseteq N$ is called $g$-independent if we have $\left.g\right|_{M}:=\{i j \in g \mid i, j \in M\}=\emptyset$, i.e. if no two players contained in $M$ are linked in $g$. Moreover, let $\mathcal{I}(g) \subseteq \mathcal{P}(N)$ denote the set of all nonempty $g$-independent subsets of $N$. The algorithm is then defined as follows.

Definition 1 (Manea (2011a)). For a given network $g$ and player set $N$, the algorithm $\mathcal{A}(g)$ provides a sequence $\left(r_{s}, x_{s}, M_{s}, L_{s}, N_{s}, g_{s}\right)_{s=1, \ldots, \bar{s}}$ which is defined recursively as follows. Let $N_{1}:=N$ and $g_{1}:=g$. For $s \geq 1$, if $N_{s}=\emptyset$ then stop and set $\bar{s}=s$. Otherwise, let

$$
r_{s}=\min _{M \subseteq N_{s}, M \in \mathcal{I}(g)} \frac{\left|L^{g_{s}}(M)\right|}{|M|}
$$

If $r_{s} \geq 1$ then stop and set $\bar{s}=s$. Otherwise, set $x_{s}=\frac{r_{s}}{1+r_{s}}$. Let $M_{s}$ be the union of all minimizers $M$ in (2). Denote $L_{s}:=L^{g_{s}}\left(M_{s}\right)$. Let $N_{s+1}:=N_{s} \backslash\left(M_{s} \cup L_{s}\right)$ and $g_{s+1}:=\left.g\right|_{N_{s+1}}$.

Given such a sequence $\left(r_{s}, x_{s}, M_{s}, L_{s}, N_{s}, g_{s}\right)_{s=1, \ldots, \bar{s}}$ being the outcome of the described algorithm $\mathcal{A}(g)$, the limit equilibrium payoff vector for this network can be determined by applying a simple rule. Note that this rather sophisticated result of Manea (2011a, Theorem 4) is absolutely fundamental for our work.

Payoff Computation (Manea (2011a)). Let $\left(r_{s}, x_{s}, M_{s}, L_{s}, N_{s}, g_{s}\right)_{s=1, \ldots, \bar{s}}$ be the outcome of $\mathcal{A}(g)$ for a given network $g$. Then the limit equilibrium payoffs are given by

$$
\begin{array}{ll}
v_{i}^{*}(g)=x_{s} & \text { for all } i \in M_{s}, s<\bar{s} \\
v_{j}^{*}(g)=1-x_{s} & \text { for all } j \in L_{s}, s<\bar{s} \\
v_{k}^{*}(g)=\frac{1}{2} & \text { for all } k \in N_{\bar{s}} .
\end{array}
$$

To see what the algorithm $\mathcal{A}(g)$ in combination with the payoff calculation rule actually does, consider a network $g$ and player set $N$. At each step $s$ the algorithm identifies the so called minimal shortage ratio $r_{s}$ among the remaining players $N_{s}$ in the network $g_{s}=\left.g\right|_{N_{s}}$, which is obtained by finding the largest $g_{s}$-independent set $M_{s}$ to minimize

$$
r_{s}=\frac{\left|L_{s}\right|}{\left|M_{s}\right|}
$$

where $L_{s}$ is the partner set of $M_{s}$. Since $M_{s}$ is $g_{s}$-independent, the only potential sources to obtain a share of the surplus for players in $M_{s}$ are the players in the partner set $L_{s}$. If $L_{s}$ is small relative to $M_{s}$, the players in $L_{s}$ are in a powerful bargaining position to extract large shares of the surplus. The algorithm then determines at each step the minimal shortage ratios to detect the players in strongest and weakest bargaining position, since they will not 
find agreements with other players. The limit equilibrium payoff of the players in $M_{s}$ is then given by $x_{s}=\frac{r_{s}}{1+r_{s}}=\frac{\left|L_{s}\right|}{\left|M_{s}\right|+\left|L_{s}\right|}<\frac{1}{2}$ while their partners in $L_{s}$ receive $1-x_{s}=\frac{\left|M_{s}\right|}{\left|M_{s}\right|+\left|L_{s}\right|}>\frac{1}{2}$ such that $x_{s}$, the share of players in $M_{s}$, is increasing in the shortage ratio. These players are then deleted from the network and the algorithm moves forward to the next step. It stops as soon as there are either no more players left or if the minimal shortage ratio is greater than or equal to one. In the latter case, the limit equilibrium payoff of all remaining players is $\frac{1}{2}$. We also illustrate how the algorithm works for the three player case in Section 3.1.

When players form the network in the first stage, they anticipate the outcome of the bargaining game. For simplicity, we also refer to this outcome as player $i$ 's payoff, i.e. the limit equilibrium payoff from the bargaining stage. However, network formation is costly such that each formed link causes costs $c>0$ for both players involved. This may be motivated as time and effort that needs to be spend in order to create a relationship. Given network $g \in G$, this implies that players bear network formation costs of $\eta_{i}(g) c$ for all $i \in N$. Thus, it is important to precisely distinguish between a player's payoff from the bargaining stage and a player's profit which we define as her payoff net of linking costs. Thus, in period $t=0$, each player $i \in N$ intends to maximize her profit

$$
u_{i}^{*}(g):=v_{i}^{*}(g)-\eta_{i}(g) c .
$$

Although only one link can generate benefits, other links may represent outside options and the bargaining outcome depends on the whole network. The forces described above then lead to conflicting interests among strategic players. Therefore, players face a trade-off when forming links: On the one hand, players benefit from own links via additional outside options. On the other hand, however, links are costly, which results in an interesting problem of network formation.

The described algorithm $\mathcal{A}(g)$ together with the previous considerations pins down payoffs and, therefore, the profit $u_{i}^{*}(g)$ of each connected player $i \in N$. It is straightforward to see that the profile of profits $u^{*}=\left(u_{i}^{*}\right)_{i \in N}$ is component-decomposable, meaning that $u_{i}^{*}(g)=$ $u_{i}^{*}\left(\left.g\right|_{C_{i}(g)}\right)$ for all players $i \in N$ and networks $g$. Here, $C_{i}(g) \subseteq N$ denotes the component of player $i$ in $g$. Thus, player $i$ 's profit is not affected by subnetworks which are induced by components not containing $i$. In particular, this implies that we can straightforwardly extend the algorithm $\mathcal{A}(g)$ to isolated players receiving a payoff of 0 since they have no bargaining partner they could generate a unit surplus with. ${ }^{8}$

We do not model strategic interaction in the network formation stage $(t=0)$ explicitly, but instead directly rely on equilibrium notions. Depending on the rules of network formation which are assumed, there are many definitions of equilibrium at hand. Here, we consider only the well-known concept of pairwise stability introduced by Jackson and Wolinsky (1996).

\footnotetext{
${ }^{8}$ Manea develops the algorithm $\mathcal{A}(g)$ under the assumption that there are no isolated players in the underlying network $g$. However, it is easy to see that it is still possible to apply the algorithm $\mathcal{A}(g)$ with $M_{1}$ consisting of all isolated players yielding $L^{g}(M)=\emptyset$ and, hence, $r_{1}=0$ implying $x_{1}=0$.
} 
Definition 2 (Pairwise Stability, Jackson and Wolinsky (1996)). Consider the player set $N$ and a profile of network utility or profit functions $\left(u_{i}\right)_{i \in N}$. Then a network $g$ is said to be pairwise stable if

(i) for all $i j \in g: u_{i}(g) \geq u_{i}(g-i j)$ and

(ii) for all $i j \notin g$ : if $u_{i}(g+i j)>u_{i}(g)$, then $u_{j}(g+i j)<u_{j}(g)$.

The implicit assumption of network formation underlying this approach to stability is that players are in control of their links; any player can unilaterally delete a given link, but to build a link, both involved players need to agree. Thus, two players will form a link if one is strictly better off and the other is not worse off, while a link is deleted, if at least one of the two involved players is strictly better off deleting the link. The networks which allow neither profitable link deletion, nor (mutually) profitable link formation are then pairwise stable. Pairwise stability is a fairly weak notion of network stability. One common refinement is to allow for multiple link deletion in (i) which is called Pairwise Nash stability. All our results are robust to this refinement. ${ }^{9}$

The analysis of our model demands to distinguish between pairwise stable networks for which the above conditions hold on a cost interval of positive length and those for which this is not the case.

Definition 3 (Generic and Nongeneric Pairwise Stability). In the considered framework with network profit function $u=u^{*}$ and linking costs $c>0$, a network $g$ is called

- generically pairwise stable if $g$ is pairwise stable for all $c^{\prime} \in(c-\epsilon, c+\epsilon)$ for some $\epsilon>0$,

- nongenerically pairwise stable if $g$ is pairwise stable but not generically pairwise stable. ${ }^{10}$

The results we derive in Section 3 will even reveal that networks can be pairwise stable at no more than a single cost level if they are not generically pairwise stable for any cost level. Thus, the notion of nongeneric pairwise stability is not robust at all with respect to changes of linking costs. We are therefore predominantly interested in generically pairwise stable networks and, in what follows, establish a complete characterization thereof.

\section{Stable Networks and Bargaining Outcomes}

To characterize stable networks, we first illustrate bargaining outcomes and profits for the three player case (Section 3.1). We use these observations to derive sufficient conditions for networks to be pairwise stable as a first step and then gradually rule out a broad range

\footnotetext{
${ }^{9}$ We decided to use the weaker notion of Pairwise Stability since the characterization results are therefore stronger.

${ }^{10}$ See e.g. Baetz (2015) for a similar definition in a setting of simultaneous choice of links and actions referred to as "generic equilibrium".
} 
of networks from being pairwise stable (Section 3.2) until we arrive at a complete characterization of generically pairwise stable networks (Section 3.3). Further, we derive the induced bargaining outcomes (Section 3.4) and elaborate on the structure of nongenerically pairwise stable networks (Section 3.5).

\subsection{Three Players}

To illustrate the model, in particular the bargaining outcomes and the implications for network formation, we first present the situation for three players. It turns out that this case already covers many important aspects of the model. Since players are ex-ante homogeneous such that their names do not matter, there are essentially four different network structures to consider (disregarding symmetries) displayed in Figure 1.

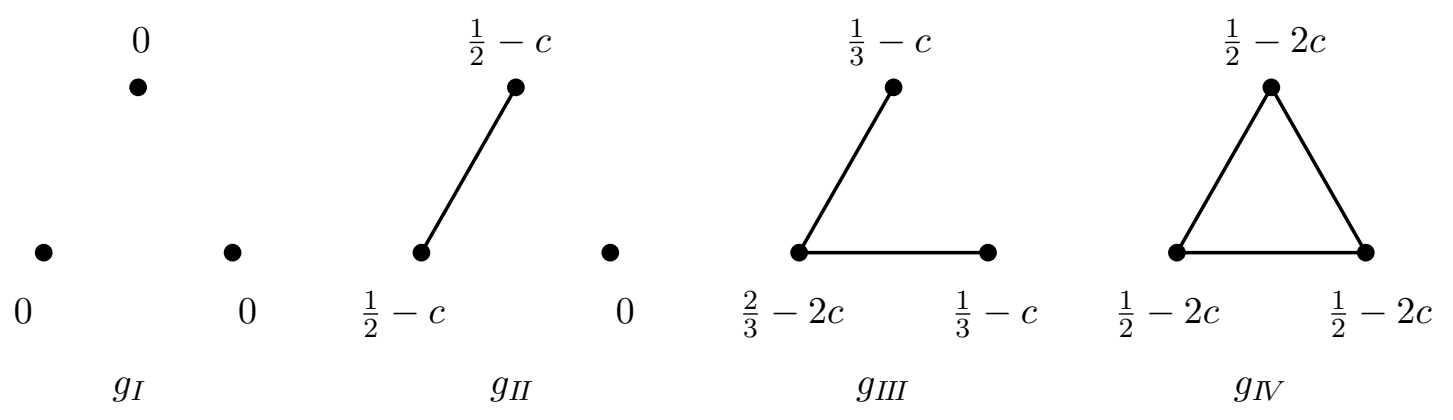

Figure 1: A sketch of the four network structures which can arise in the case $n=3$ with induced profits

Limit equilibrium payoffs can be calculated as follows. Players who are not connected always receive payoff 0 from bargaining, but also do not incur any network formation costs. In the one link network $g_{I I}$, the connected players are in symmetric positions implying limit equilibrium payoffs of $\frac{1}{2}$ each, which can also be obtained by applying Manea's algorithm (Definition 1) since the shortage ratio is unity. Because both players have formed one link in this network, their profit in the network formation stage is $u_{i}^{*}\left(g_{I I}\right)=\frac{1}{2}-c$. In contrast, players in $g_{I I I}$ are in asymmetric network positions. The central player acts as a monopolist to the two peripheral players who depend on the central player to come to an agreement. Therefore, the two peripheral players are the largest $g$-independent set $M_{1}$ minimizing the shortage ratio which can be calculated to be $r_{1}=\left|L_{1}\right| /\left|M_{1}\right|=\frac{1}{2}$. Thus, limit equilibrium payoffs are $v_{i}^{*}\left(g_{I I I}\right)=x_{1}=\frac{r_{1}}{1+r_{1}}=\frac{1}{3}$ for the peripheral players $i \in M_{1}$, implying $v_{j}^{*}\left(g_{I I I}\right)=1-x_{1}=\frac{2}{3}$ for the central player $j$. Subtracting link formation cost, we get for profits $u_{i}^{*}\left(g_{I I I}\right)=\frac{1}{3}-c$ and $u_{j}^{*}\left(g_{I I I}\right)=\frac{2}{3}-2 c$, respectively. Finally in the complete network $g_{I V}$, all players are in symmetric positions. The only $g$-independent sets are singleton players for which the shortage ratio exceeds unity, implying that limit equilibrium payoffs equal $\frac{1}{2}$ with linking costs $2 c$, yielding profits of $u_{i}^{*}\left(g_{I V}\right)=\frac{1}{2}-2 c$, each.

Considering network formation in the first stage, we can calculate the marginal profits of each link for the involved players. We have $u_{i}^{*}\left(g_{I I}\right)-u_{i}^{*}\left(g_{I}\right)=\frac{1}{2}-c$ for connected players $i$ in $g_{I I}$, meaning that players have a strict incentive to form a link in the empty network, if $c<\frac{1}{2}$. Thus, the empty network is pairwise stable if and only if $c \geq \frac{1}{2}$. Further, we get 
$u_{j}^{*}\left(g_{I I I}\right)-u_{j}^{*}\left(g_{I I}\right)=\frac{1}{6}-c$ for the central player $j$ in $g_{I I I}$ implying that the connected players in $g_{I I}$ have a weak incentive to form a link with the isolated player if $c \leq \frac{1}{6}$. Since for the isolated player $k$ in $g_{I I}$ marginal utility $u_{k}^{*}\left(g_{I I I}\right)-u_{k}^{*}\left(g_{I I}\right)=\frac{1}{3}-c$ is strictly positive if $c \leq \frac{1}{6}$, network $g_{I I}$ is pairwise stable if and only if $c \in\left(\frac{1}{6}, \frac{1}{2}\right]$. Finally, we get $u_{i}^{*}\left(g_{I V}\right)-u_{i}^{*}\left(g_{I I I}\right)=\frac{1}{6}-c$ for the peripheral players $i$ in $g_{I I I}$. Thus, in order for the peripheral players in $g_{I I I}$ not to have a strict incentive to form the link, we must have $c \geq \frac{1}{6}$, while for symmetric reasons to the considerations above, cost must satisfy $c \leq \frac{1}{6}$ for the central player in $g_{I I I}$ not to have an incentive to delete the link. Hence, the network $g_{I I I}$ is only pairwise stable for a single cost value $c=\frac{1}{6}$ which means that $g_{I I I}$ is nongenerically pairwise stable according to Definition 3 . Finally, the network $g_{I V}$, which is a circle of odd size in this three player example, is pairwise stable for $c \in\left[0, \frac{1}{6}\right]$.

The example already conveys some of the main insights, as only the empty network $\left(g_{I}\right)$ or networks such that any nonsingleton component is either a separated pair $\left(g_{I I}\right)$ or an odd circle $\left(g_{V}\right)$ can be generically pairwise stable. Payoff from bargaining in generically pairwise stable networks is always such that nonisolated players receive homogeneous payoffs of $\frac{1}{2} \cdot{ }^{11}$ Other structures (here: $g_{I I I}$ ) may arise as nongenerically pairwise stable, allowing for heterogeneous bargaining outcomes.

\subsection{Necessary and Sufficient Conditions for Networks to Be Pairwise Stable}

The insights from the three player example can be generalized to an arbitrary number of players $n$. We first show that networks for which each component has exactly the observed characteristics from the three player case are pairwise stable for $n \geq 3$.

Theorem 1 (Sufficient Conditions for Networks to Be Pairwise Stable).

(i) The empty network is pairwise stable if $c \geq \frac{1}{2}$.

(ii) A network which is a union of separated pairs and not more than one isolated player is pairwise stable if $c \in\left(\frac{1}{6}, \frac{1}{2}\right] .{ }^{12}$ Additionally, if $c=\frac{1}{2}$, then there can exist more than one isolated player.

(iii) A network which is a union of odd circles with at most $\frac{1}{2 c}$ players and either separated pairs or at most one isolated player is pairwise stable if $c \in\left(0, \frac{1}{6}\right] .{ }^{13}$ Additionally, if $c=\frac{1}{6}$ and given that there is no isolated player, then there can also exist lines of length three in a pairwise stable network. ${ }^{14}$

\footnotetext{
${ }^{11}$ Note the relation to equitable networks (Manea, 2011a) which are defined as the networks giving rise to symmetric limit equilibrium payoffs of $\frac{1}{2}$ in the bargaining stage.

${ }^{12}$ A separated pair denotes a subnetwork induced by a two-player component.

${ }^{13}$ Here, the union can be exclusive such that a network consisting only of separated pairs or only of (some of the permissible) odd circles is pairwise stable. A circle with $m$ players or a $m$-player circle is induced by a component with cardinality $m \geq 3$ such that all players have exactly 2 links. It is called odd if its cardinality is an odd number.

${ }^{14} \mathrm{~A}$ line of length $m \geq 3$ denotes a subnetwork induced by a $m$-player component which can be transformed to a $m$-player circle by adding one link.
} 
The formal proof of this theorem, like all other more evolved and lengthy proofs, is provided in the appendix. Recalling the three player case, the intuition is quite simple, however. While incentives to form links observed in the three player case carry over to the $n$ player case, we establish that links across these components are not profitable for the involved players which then explains the structure of the stable networks. Therefore, any network such that each component has the same form as one of the stable structures in the three player case is pairwise stable. Additionally, components can be odd circles of larger size for small cost of link formation. The intuition is that link deletion in an odd circle leaves the two involved players as the end of an odd line. The payoff loss due to link deletion decreases with the size of the odd circle such that large circles can only be stable for small costs of link formation.

Theorem 1 above lists some stable networks for the specified cost ranges. Therefore as a byproduct of Theorem 1, we get existence of a pairwise stable network for any value of costs $c$. In priciple, more networks than listed in Theorem 1 may be pairwise stable. In the remainder of this section, however, we derive necessary conditions that show that the networks which are not listed in Theorem 1 can at most be nongenerically pairwise stable. Hence, we show that the networks in Theorem 1 which are stable for a cost interval of positive length characterize the set of generically pairwise stable networks completely. As a first step, we can use observations from the proof of Theorem 1 and deductions from the three player case to rule out some particular network structures from being pairwise stable.

Corollary 1. A network cannot be pairwise stable if it contains

(i) more than one isolated player while $c<\frac{1}{2}$,

(ii) a separated pair while $c>\frac{1}{2}$,

(iii) a line of length three while $c \neq \frac{1}{6}$,

(iv) an odd circle with more than $\frac{1}{2 c}$ members, ${ }^{15}$

$(v)$ an isolated player combined with a separated pair or a line of length three while $c \leq \frac{1}{6}$.

Statements (i)-(iv) as well as the first part of Statement (v) of Corollary 1 follow immediately from what we learned in the three-player case and the proof of Theorem 1 . To see that an isolated player and a line of length three cannot coexist in a pairwise stable network recall from Section 3.1 that an isolated player receives profit 0 while the two peripheral players in a line of length three receive $\frac{1}{3}-c$. If one of these players connects to an isolated player, then the algorithm $\mathcal{A}(\cdot)$ yields that all players in the new component receive a payoff of $\frac{1}{2}$. Thus, the isolated player benefits strictly while the peripheral player in the line benefits at least weakly from the formation of this link for $c \leq \frac{1}{6}$.

\footnotetext{
${ }^{15}$ In particular, this means that there can be no odd circles at all in pairwise stable networks as long as $c>\frac{1}{6}$.
} 
In order to find necessary conditions for networks to be pairwise stable, we proceed with a simple observation. Consider some network $g \in G$. Recall from Manea's analysis that a link $i j \in g$ can only lead to an agreement if $v_{i}^{* \delta}(g)+v_{j}^{* \delta}(g) \leq 1$ holds for $\delta$ large enough. The links $i j \in g$ such that above does not hold are called disagreement links. From a network formation perspective, intuition then says that disagreement links are irrelevant regarding payoffs while being costly and therefore should not form. Since the limit equilibrium network $g^{*}$ of a network $g$ is defined in Manea (2011a) as the network obtained by deleting all disagreement links from $g$ for $\delta \rightarrow 1$, above intuition is confirmed in the following result.

Proposition 1. If $g$ is pairwise stable, then $g=g^{*}$ and, hence, $v_{i}^{*}(g)+v_{j}^{*}(g)=1 \forall i j \in g$.

Proposition 1 will prove to be of great importance for showing that the generically pairwise stable networks given in Theorem 1 are the only generically pairwise stable networks. The subsequent results on necessary conditions for a network to be pairwise stable make heavily use of the fact that pairwise stable networks cannot contain disagreement links and that therefore payoffs of connected players must add up to 1.

First, let us observe the implications of Proposition 1 for components in which players receive homogeneous payoffs. In such a component $C$, two players $i, j \in C$ with $i j \in g$ then must receive $v_{i}^{*}(g)=\frac{1}{2}=v_{j}^{*}(g)$. Recall that the generically pairwise stable networks in Theorem 1 are all such that nonisolated players receive homogeneous payoffs of $\frac{1}{2}$ in the bargaining stage as these players are either part of a separated pair or an odd circle. We show in the following that these are the only possible substructures of pairwise stable networks giving rise to equal payoffs of $\frac{1}{2}$. To do so, define the subset $\tilde{N}(g):=\left\{i \in N \mid v_{i}^{*}(g)=\frac{1}{2}\right\}$ as the subset of players receiving equal payoffs of $\frac{1}{2}$.

Theorem 2 (Necessary Conditions for Networks to Be Pairwise Stable - Part 1/2). If a network $g$ is pairwise stable, then for any component $C$ of $g$ it holds that $C \cap \tilde{N}(g) \in\{\emptyset, C\}$. If $C \cap \tilde{N}(g)=C$, then it must be a separated pair or an odd circle.

The proof, which is again relegated to the appendix, makes use of Proposition 1 and Manea (2011a, Theorem 5). Because by Proposition 1, payoffs of connected players must add up to one, we get that players receiving a payoff of $\frac{1}{2}$ can only be connected to other players receiving the same payoff showing the first part of the statement. Moreover, since the network $\left.g\right|_{\tilde{N}(g)}$ is equitable by definition, we can apply Manea (2011a, Theorem 5) where it is shown that a network is equitable if and only if it has an edge cover $g^{\prime}$ composed of separated pairs and odd circles. A network $g^{\prime}$ is said to be an edge cover of $\left.g\right|_{\tilde{N}(g)}$ if it fulfills $\left.g^{\prime} \subseteq g\right|_{\tilde{N}(g)}$ and no player in $\tilde{N}(g)$ is isolated in $g^{\prime}$. Applying Proposition 1 again, this implies that any player in $\tilde{N}(g)$ has an incentive to delete each of her links not contained in $g^{\prime}$.

Though statements differ, note that Theorem 2 above is in line with Manea (2011b, Theorem 1(ii)). The latter establishes that for zero linking costs a network is pairwise stable if and only if it is equitable and therefore allows for a larger class of pairwise stable (equitable) networks. Among them, for instance, can be networks containing circles or lines 
of even length or completely connected components of arbitrary size as subnetworks. Many of these can be ruled out when linking costs are introduced by Theorem 2 .

Moreover, as we have seen in Figure 1 and Theorem 1, our model with linking costs also allows for nonequitable structures to be pairwise stable, such as networks containing isolated players or the line of length three as a subnetwork. The reasons for the appearance of these nonequitable structures are twofold: While isolated players can be left out due to the fact that nonisolated players cannot improve their bargaining payoff by connecting to an isolated player, we have so far observed nonequitable, component induced subnetworks only in nongenerically pairwise stable networks. Thus, the networks which induce heterogeneous payoffs within a component remain to be examined. Straightforwardly, as an implication of Proposition 1 and by applying the payoff computation rule, we get the following condition on payoffs for players in these nonequitable components. ${ }^{16}$

Corollary 2. If a network $g$ is pairwise stable, then for any nonequitable component $C$ of $g$, there exists a unique partition $M \dot{\cup} L=C$ with $|M|>|L|$ and $\left.g\right|_{M}=\left.g\right|_{L}=\emptyset$ such that payoffs are given by

$$
\begin{array}{ll}
v_{i}^{*}(g)=x \quad \text { for all } i \in M & \text { and } \\
v_{j}^{*}(g)=1-x & \text { for all } j \in L,
\end{array}
$$

where $x=\frac{|L|}{|M|+|L|}$.

If a pairwise stable network contains a nonequitable component, then the component induced subnetwork and the payoffs of the involved players must be of special structure: The component induced subnetwork has to be bipartite since by Proposition 1 payoffs of connected players must add up to one, implying that players receiving the same payoff within a nonequitable component cannot be neighbors. For given cardinalities of both sides of the bipartite subnetwork, the admissible payoffs can exactly be calculated by applying Manea's algorithm $\mathcal{A}(g)$. This has important consequences for the existence of such components when we take into account cost of link formation. It turns out that, given link formation cost $c$, only one payoff pair is feasible in a pairwise stable network, and therefore the structure of the induced subnetworks is crucially restricted.

Theorem 3 (Necessary Conditions for Networks to Be Pairwise Stable - Part 2/2). If a network $g$ is pairwise stable, then for any nonequitable component $C$ of $g$, players obtain payoffs of either $x \in\left(0, \frac{1}{2}\right)$ or $1-x \in\left(\frac{1}{2}, 1\right)$ that satisfy

$$
x+c=\frac{1}{2} .
$$

The proof uses two lemmas which are of independent interest. Therefore, the Lemmas

\footnotetext{
${ }^{16}$ We speak of a (non)equitable component $C$ if the component induced subnetwork $\left.g\right|_{C}$ is (non)equitable.
} 
are stated below while the proof of them is relegated to the appendix. Given these Lemmas, the proof of Theorem 3 is quite intuitive and shown below the respective Lemmas 1 and 2 .

In each nonequitable component of a pairwise stable network, there exist some players receiving payoff $x<\frac{1}{2}$ and some players receiving payoff $1-x>\frac{1}{2}$ by Corollary 2. Lemma 1 then deals with players receiving a payoff $x<\frac{1}{2}$. It is shown that if two such players connect or one such player connects to an isolated player, then they each receive a payoff equal to $\frac{1}{2}$. Note that for both Lemmas 1 and 2 , we have in mind that in a pairwise stable network restricted to a nonequitable component, the algorithm $\mathcal{A}(g)$ stops after one step. This is due to the fact that according to Manea (2011a, Proposition 3), the sequence of minimal shortage ratios provided by the algorithm in Definition 1 is strictly increasing for any network. Since only two distinct payoffs can occur when restricted to a component of a pairwise stable network by Corollary 2, we cannot have more than one iteration of the algorithm.

Lemma 1. If a network $g$ is pairwise stable and the algorithm $\mathcal{A}(g)$ provides $\left(r_{1}, x_{1}, M_{1}, L_{1}\right.$, $\left.N_{1}, g_{1}\right)$, i.e. $\bar{s}=1$, such that $r_{1} \in(0,1)$, then for all $i, j \in M_{1}$ it is

$$
v_{i}^{*}(g+i j)=v_{j}^{*}(g+i j)=\frac{1}{2} .
$$

Further, if the player set $N=\{1, \ldots, n\}$ is extended by an isolated player $n+1$ such that the network $g$ remains unchanged, it similarly is $v_{i}^{*}(g+i(n+1))=v_{n+1}^{*}(g+i(n+1))=\frac{1}{2}$.

Having characterized the marginal payoffs of players receiving payoff $x<\frac{1}{2}$, Lemma 2 then deals with players in nonequitable components of a pairwise stable network receiving payoffs $1-x>\frac{1}{2}$. It is shown that it is not possible to reduce these players' payoffs below $\frac{1}{2}$ by the deletion of a link. At the same time, the payoff of players receiving $x<\frac{1}{2}$ cannot be increased to more than $\frac{1}{2}$ by link deletion.

Lemma 2. If a network $g$ is pairwise stable and the algorithm $\mathcal{A}(g)$ provides $\left(r_{1}, x_{1}, M_{1}, L_{1}\right.$, $\left.N_{1}, g_{1}\right)$, i.e. $\bar{s}=1$, such that $r_{1} \in(0,1)$, then for all $j \in L_{1}, i \in M_{1}$ and $k l \in g$ it is

$$
v_{j}^{*}(g-k l) \geq \frac{1}{2} \geq v_{i}^{*}(g-k l) .
$$

The proofs of these lemmas are somewhat lengthy and as usual provided in the appendix. In both cases we show that, if the respective statement were not true, then this would imply that the player set is infinite. To arrive at this contradiction, we make use of an additional, rather technical lemma which we also provide in the appendix (see Lemma 3). Based on these lemmas, the proof of the theorem can be obtained straightforwardly.

Proof of Theorem 3. Let $g$ be a pairwise stable network inducing heterogeneous payoffs within some component $C \subseteq N$. Let $g^{\prime}:=\left.g\right|_{C}$. According to Corollary 2, the algorithm $\mathcal{A}\left(g^{\prime}\right)$ (with $N_{1}=C$ ) has to stop after the first step, i.e. $\bar{s}^{\prime}=1$. Let $\left(r_{1}^{\prime}, x_{1}^{\prime}, M_{1}^{\prime}, L_{1}^{\prime}, N_{1}^{\prime}, g_{1}^{\prime}\right)$ be the outcome of $\mathcal{A}\left(g^{\prime}\right)$ and $i \in M_{1}^{\prime}, j \in L_{1}^{\prime}$. Then, $v_{i}^{*}(g)=x_{1}^{\prime}=\frac{\left|L_{1}^{\prime}\right|}{\left|M_{1}^{\prime}\right|+\left|L_{1}^{\prime}\right|} \in\left(0, \frac{1}{2}\right)$ and 
$v_{j}^{*}(g)=1-x_{1}^{\prime}=\frac{\left|M_{1}^{\prime}\right|}{\left|M_{1}^{\prime}\right|+\left|L_{1}^{\prime}\right|} \in\left(\frac{1}{2}, 1\right)$. For $g$ to be pairwise stable, it must not be mutually profitable for $i, k \in M_{1}^{\prime}$ to create a link, which by Lemma 1 implies that

$$
x_{1}^{\prime}-\eta_{i}\left(g^{\prime}\right) c \geq \frac{1}{2}-\left(\eta_{i}\left(g^{\prime}\right)+1\right) c \quad \Leftrightarrow \quad x_{1}^{\prime}+c \geq \frac{1}{2} .
$$

Similarly, it must not be profitable to delete a link for $j \in L_{1}^{\prime}$, which by Lemma 2 implies that

$$
\left(1-x_{1}^{\prime}\right)-\eta_{j}\left(g^{\prime}\right) c \geq \frac{1}{2}-\left(\eta_{j}\left(g^{\prime}\right)-1\right) c \quad \Leftrightarrow \quad x_{1}^{\prime}+c \leq \frac{1}{2} .
$$

Thus, $v_{i}^{*}(g)=x_{1}^{\prime}=\frac{1}{2}-c$ and $v_{j}^{*}(g)=1-x_{1}^{\prime}=\frac{1}{2}+c$. Obviously, this has to hold for all components of $g$ in which players receive heterogeneous payoffs implying the result by component decomposability.

Because by Lemma 1 players in unfavorable bargaining positions in nonequitable components gain exactly $\frac{1}{2}-x$ if they connect to each other, cost must exceed the marginal profit of such a link for the network to be pairwise stable. On the other hand, since by Lemma 2 players with highest bargaining power in such components do not lose more than $1-x-\frac{1}{2}$ when deleting a link, cost must be below the marginal profit of such a link for the network to be pairwise stable. Hence, payoffs in nonequitable components and thereby also the structure of these are uniquely determined by cost $c$. Notice also, by considering the limit case $c \rightarrow 0$, that Theorem 3 is in line with Manea (2011b, Theorem 1) stating that, for zero linking costs, any pairwise stable network must be equitable.

\subsection{A Complete Characterization of Generically Pairwise Stable Networks}

We are now in a position to put all our results on necessary conditions for pairwise stable networks together to find that those networks in Theorem 1 which are stable for a cost interval of positive length are the only networks which can be generically pairwise stable. Therefore we arrive at a complete characterization of generically pairwise stable networks.

Corollary 3 (Complete Characterization). A network is generically pairwise stable if and only if it is either

(i) the empty network, or

(ii) a union of separated pairs, odd circles and at most one isolated player such that an isolated player only appears if either all other components are separated pairs or all other components are odd circles.

In particular, no network other than the ones provided in Theorem 1 is generically pairwise stable for the respective cost level.

The appearance of pairs and odd circles and the admissible size of odd circles depend on the cost of link formation given in Theorem 1. Note that for any given size of an odd 
circle, it is always possible to find a cost interval of positive length such that the network is pairwise stable by Theorem 1 . To see that no other networks can be generically pairwise stable, note that by Theorem 2 any equitable component must be an odd circle or a separated pair. Corollary 1 together with Theorem 2 then implies that any pairwise stable network not captured by Theorem 1 must contain a nonequitable component.

For pairwise stable networks containing nonequitable components, the payoff distribution of players within the nonequitable component is uniquely determined by the exogenously given cost level $c$, see Theorem 3. At the same time, the component induced subnetwork must be bipartite such the sizes of both sides of the bipartite network are also uniquely by $c$ due to Corollary 2. Hence, a perturbation of $c$ yields different conditions for these group sizes, implying that if a pairwise stable network contains a nonequitable component, then it is pairwise stable only for a single cost value. In other words, all pairwise stable networks containing nonequitable components are nongenerically pairwise stable. Hence, the networks in Corollary 3 completely characterize the set of generically pairwise stable networks.

Beyond that, given the crucial cost level $c=\frac{1}{2}-x$, it is not at all clear that a network in which each player receives a payoff of $x \in\left(0, \frac{1}{2}\right)$ or $1-x$ is actually pairwise stable. First, $c$ must be a rational number, as otherwise the shortage ratio cannot meet the condition on payoffs. Rational numbers also put restrictive conditions on the number of players $n$ for existence. Hence, the emergence of networks with nonequitable components is very unlikely. Second, nongenerically pairwise stable networks are less stable from another perspective: Any two players on the short side of the market receiving payoff $x$ must be indifferent between leaving the network unchanged and adding a mutual link (see Lemma 1). Also, any player receiving a payoff of $1-x$ must be indifferent between keeping and deleting any one link (see Lemma 2). Therefore, networks which do not have the structure as given in Corollary 3, are neither robust to perturbation of the cost level nor are they robust to perturbation of best responses in network formation. In that sense, generically pairwise stable networks are the likely outcome of a network formation game prior to a Manea (2011a) bargaining game.

\subsection{Induced Bargaining Outcomes}

In the previous sections, we have derived necessary conditions for pairwise stability and completely characterized generically pariwise stable networks. Although we already discussed implications for the bargaining outcome, it is worthwhile to summarize them. As our second main result, we straightforwardly conclude that payoffs and profits induced by (generically) pairwise stable networks are in general highly but not completely homogeneous.

Corollary 4 (Limited Outcome Diversity). Consider a pairwise stable network $g$.

(i) If $g$ is generically pairwise stable for $c>\frac{1}{2}$, then all players receive $v_{i}^{*}(g)=u_{i}^{*}(g)=0$.

(ii) If $g$ is generically pairwise stable for $c<\frac{1}{2}$, then all nonisolated players receive payoff $v_{i}^{*}(g)=\frac{1}{2}$ while there can be at most one isolated player receiving a payoff $v_{j}^{*}(g)=0$. Profits satisfy $u_{i}^{*}(g) \in P(g)$ for all $i \in N$ where $P(g) \subset\left\{0, \frac{1}{2}-2 c, \frac{1}{2}-c\right\}$ with $|P(g)| \leq$ 
2. Players in odd circles obtain $u_{i}^{*}(g)=\frac{1}{2}-2 c$ while players in separated pairs get $u_{i}^{*}(g)=\frac{1}{2}-c$ and isolated players receive $u_{i}^{*}(g)=0$

(iii) If $g$ is nongenerically pairwise stable, bargaining payoffs of players are such that either $v_{i}^{*}(g) \in\left\{\frac{1}{2}-c, \frac{1}{2}, \frac{1}{2}+c\right\}$, or $v_{i}^{*}(g) \in\left\{0, \frac{1}{2}\right\}$ for all $i \in N$. The former case of nonequitable components only exists if $c \in\left(0, \frac{1}{4}\right]$.

The first and second part of Corollary 4 follow straightforwardly from our complete characterization of generically pairwise stable networks (see Corollary 3) and the fact that players in odd circles and separated pairs receive payoff $v_{i}^{*}(g)=\frac{1}{2}$. It implies that in the most likely result of network formation, i.e. in generically pairwise stable networks, all players (except possibly one) receive the same payoff: in the empty network all receive $v_{i}^{*}(g)=0$ while in all other generically pairwise stable networks at most one gets $v_{i}^{*}(g)=0$ while all others receive $v_{i}^{*}(g)=\frac{1}{2}$ in the bargaining stage. Profits in the network formation stage may however differ. While players in odd circles maintain two links, players in separated pairs have only formed one link and therefore only incur half the linking costs while receiving the same payoff from bargaining. In that sense, separated pairs are the efficient outcome and odd circles only form because of the myopic nature of link formation assumed in the notion of pairwise stability.

Additionally to these considerations, nonequitable components can exist as part of nongenerically pairwise stable networks allowing for slightly more diverse bargaining outcomes. Corollary 4(iii) further states that isolated players receiving payoff 0 cannot coexist with nonequitable components in (nongenerically) pairwise stable network. The intuition is that in such a structure, an isolated player has a strict incentive to add a link while a player in a nonequitable component receiving $v_{i}^{*}(g)=\frac{1}{2}-c$ is indifferent between connecting to the isolated player or not (recall Lemma 1). A similar intuition is discussed for part (v) of Corollary 1. Moreover, Corollary 4(iii) states that nonequitable components can only exist if $c \leq \frac{1}{4}$. The reason is that players in nonequitable components receiving payoffs $v_{i}^{*}(g)=\frac{1}{2}-c$ have at least one link which is costly. Deleting a link to $j \in N_{i}(g)$ would not lower payoffs from bargaining below 0 , i.e. $v_{i}^{*}(g-i j) \geq 0$, meaning that marginal profit of the link $i j$ is at most $\frac{1}{2}-c-c$ which is negative for $c>\frac{1}{4}$ contradicting pairwise stability.

Taken together, we have that the diversity of possible bargaining outcomes gets narrowed down substantially compared to the work of Manea (2011a) if one considers a preceding stage of costly strategic network formation. If all networks were possible, one could obtain any rational number from the interval $[0,1)$ as a payoff induced by an appropriate network on a sufficiently large player set in Manea's basic framework with $\delta \rightarrow 1 .{ }^{17}$ Strategic network formation rules out most of these networks. However, compared to Manea (2011b, Theorem 1) a larger diversity of bargaining outcomes is possible when link formation is costly.

\footnotetext{
${ }^{17}$ For the rational number $\frac{n^{\prime}}{n^{\prime \prime}} \in[0,1)$ with $n^{\prime}, n^{\prime \prime} \in \mathbb{N}$, consider the player set $N$ with $n=n^{\prime \prime}$ and the complete bipartite network with $n^{\prime}$ players on one side and $n^{\prime \prime}-n^{\prime}$ players on the other side. Then the algorithm $\mathcal{A}(\cdot)$ yields payoffs $\frac{n^{\prime \prime}-n^{\prime}}{n^{\prime \prime}}$ and $\frac{n^{\prime}}{n^{\prime \prime}}$.
} 


\subsection{Nongenerically Pairwise Stable Networks}

Although nongenerically pairwise stable networks do not seem to be a very likely outcome when network formation is considered in the first stage, we complete our analysis by studying these networks. First, note that for $c>\frac{1}{2}$ no nongenerically pairwise stable network exists as the unique pairwise stable network is the empty network which is generically pairwise stable. ${ }^{18}$ Some nongenerically pairwise stable networks are already given in Theorem 1. For $c=\frac{1}{2}$ more than one isolated players and separated pairs may coexist since players are indifferent between forming a link or not. For $c<\frac{1}{2}$, a nongenerically pairwise stable network $g$ must contain at least one nonequitable components by our previous results. Each such nonequitable component $C$ must be bipartite, i.e. consist of two $g$-independent sets $M$ and $L$ with $M \dot{\cup} L=C$ satisfying $\frac{|L|}{|M|+|L|}=\frac{1}{2}-c$, see Theorem 3. For the derivation of this, we only used the fact that in a pairwise stable network, both link addition between two players in the set $M$ and link deletion by a player in $L$ cannot be profitable. Considering both the incentives of players in $M$ and $L$ for link deletion in more detail, leads to further restrictions on the structure of nonequitable components. To be more precise, we establish that a nonequitable component cannot be a $t_{r e e}{ }^{19}$ (except for the three player tree considered in Theorem 1), cannot contain a cycle ${ }^{20}$ with a cut-player on the strong side of the market $L$, and has to satisfy further conditions as stated below. For a given network $g$, a player $k \in N$ is called cut-player if $\left.g\right|_{N \backslash\{k\}}$ has more components than $g \cdot{ }^{21}$

Proposition 2. If a network $g$ is nongenerically pairwise stable and $c<\frac{1}{2}$, then $g$ contains at least one nonequitable component of at least three players. Every such component $C$ is bipartite and consists of $g$-independent sets $M, L \subset C$ with $M \dot{\cup} L=C$, satisfying $\frac{|L|}{|M|+|L|}=$ $\frac{1}{2}-c$ such that the following has to hold:

(i) C cannot be a tree of more than three players.

(ii) C cannot contain a cycle which encompasses a cut-player in $L$.

(iii) $|M| \leq 3|L|$.

(iv) For all $i \in M, j \in L$ with $i j \in g$ there exists $M^{\prime} \subset M$ and corresponding partner set $L^{\prime}:=L^{g-i j}\left(M^{\prime}\right) \subset L$ in $g-i j$ with $i \in M^{\prime}$ and $M^{\prime} \cap N_{j}(g)=\{i\}$ such that for $m^{\prime}=\left|M^{\prime}\right|$ and $l^{\prime}=\left|L^{\prime}\right|$, we have

$$
c \leq \min \left\{\frac{1}{2}\left(\frac{1}{2}-\frac{l^{\prime}}{l^{\prime}+m^{\prime}}\right), \frac{m^{\prime}}{\left(m^{\prime}+l^{\prime}\right)^{2}+\left(m^{\prime}+l^{\prime}\right)}\right\} .
$$

\footnotetext{
${ }^{18}$ To see this most easily, note that for any pairwise stable network $g$ and a player $i$ receiving $v_{i}^{*}(g)>\frac{1}{2}$, marginal payoff of any link satisfies $v_{i}^{*}(g)-v_{i}^{*}(g-i j)<\frac{1}{2}$ by Lemma 2 . Thus, any link will be deleted as cost of the link exceeds any additional payoff.

${ }^{19} \mathrm{~A}$ tree denotes a component-induced subnetwork which is minimally connected.

${ }^{20} \mathrm{~A}$ cycle is a path from a player $i$ to itself.

${ }^{21}$ This notation comes from graph theory where vertices of that kind are typically called "cut-vertices" (see e.g. West, 2001). For instance, each player contained in a component which induces a tree and having more than one link is a cut-player.
} 
The result restricts the set of nonequitable components that can be part of a pairwise stable network. Except for the line of three players (see Figure 1, network $g_{I I I}$ ), no other trees can be component induced. Hence, all other nonequitable components of pairwise stable networks have to contain cycles. By Proposition 2(ii), such a cycle cannot encompass a cut-player in $L$. Thus, there cannot exist a loose-end player $i \in M$ whose single neighbor is part of a cycle. ${ }^{22}$ Proposition 2(ii), however, is more general than just excluding loose-end players in $M$. Some examples of network structures that cannot be pairwise stable due to Proposition 2(ii) are illustrated in Figure 2 where $g_{V}$ contains a loose-end player and in $g_{V I}$ the central player is a cut-player.

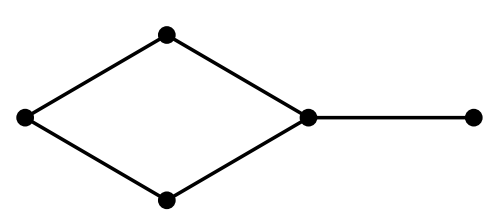

$g_{V}$

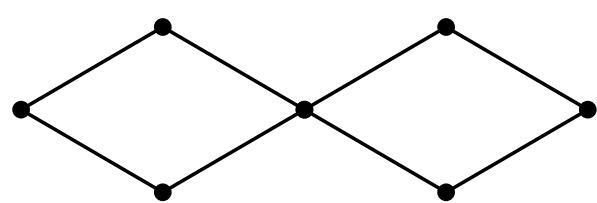

$g_{V I}$

Figure 2: A sketch of networks which cannot be pairwise stable according to Proposition 2(ii)

Further, Proposition 2(iii) establishes that too unbalanced bipartite structures with respect to the sizes of the independent sets cannot be pairwise stable. The rather unintuitive part (iv) simply implies that the bipartite component cannot have too many links. This follows from the fact that every link contained in the bipartite component must yield positive marginal payoff exceeding the cost of link formation. That means that after the deletion of a link $i j \in g$, player $i \in M$ (and also $j$ ) incurs a payoff loss such that the algorithm $\mathcal{A}(g-i j)$ must detect a smaller shortage ratio $\frac{L^{\prime}}{M^{\prime}}<\frac{L}{M}$ in the first step with $i \in M^{\prime}$ and $j \notin L^{\prime}$ since nothing else has changed. From $j \notin L^{\prime}$ it then follows that $N_{j}(g-i j) \cap M^{\prime}=\emptyset$. The condition on $c$ in Proposition 2(iv) ensures that payoff loss due to link deletion is large enough. Two networks which are captured by Proposition 2(iv) and can therefore not be pairwise stable are pictured in Figure 3. To see this, pick a link $i j \in g_{V I I}$, and consider some set $M^{\prime} \subset M$ with $i \in M^{\prime}$. Then for $M^{\prime} \neq\{i\}$ we have $M^{\prime} \cap N_{j}(g) \neq\{i\}$ and for $M^{\prime}=\{i\}$ we get $\frac{1}{2}\left(\frac{1}{2}-\frac{l^{\prime}}{l^{\prime}+m^{\prime}}\right)=0$ and hence the network cannot be pairwise stable. Similar considerations hold for some links in $g_{V I I I}$ as it contains $g_{V I I}$.

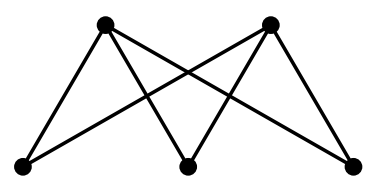

$g_{V I I}$

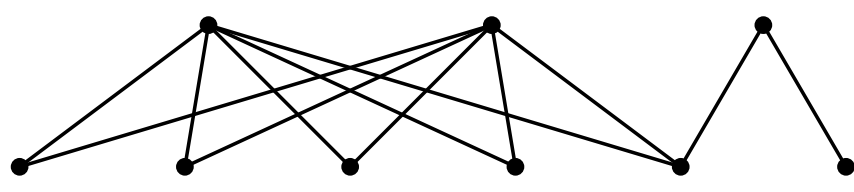

$g_{V I I I}$

Figure 3: A sketch of networks which cannot be pairwise stable according to Proposition 2(iv)

\footnotetext{
${ }^{22}$ Also note that no player $j \in L$ can be a loose-end since otherwise $L^{-j}:=L \backslash\{j\}$ and $M^{-j}:=M \backslash N_{j}(g)$ result in smaller shortage ratio $\frac{L^{-j}}{M^{-j}}<\frac{L}{M}$.
} 
Though a further generalization is not reached here, Proposition 2 rules out many networks from being even nongenerically pairwise stable. It remains as a conjecture that the only nongenerically pairwise stable networks inducing heterogeneous payoffs within a component are those containing a line of length three at cost level $c=\frac{1}{6}$.

\section{Discussion and Conclusion}

With this paper we contribute to the study of endogenously generated market structures. To model market interaction we employ Manea's (2011a) seminal approach to bargaining on networks. While Manea (2011a) characterizes bargaining outcomes for any exogenously given network, the focus of our paper is to determine which networks actually arise when incentives to form costly links are determined by the bargaining game. Thereby we are able to draw conclusions about the induced bargaining outcome when ex ante homogeneous players form a network resulting in possibly heterogeneous bargaining positions.

Although the bargaining outcome in Manea (2011a) depends in a nontrivial way on the network structure and can only be computed via an algorithm, we arrive at an analytically tractable model of strategic network formation. Considering infinitely patient players, we completely characterize the set of generically pairwise stable networks. These are the most likely outcome of network formation as nongenerically pairwise stable networks are neither robust to changes in costs nor to perturbation of best replies.

Nonempty generically pairwise stable networks consist of specific unions of separated pairs, odd circles, and at most one isolated player, where the particular structure depends on the level of linking costs as shown in Theorem 1. The induced bargaining outcomes are homogeneous since all nonisolated players receive equal payoff of $\frac{1}{2}$ in the bargaining stage. In this sense the networks restricted to nonisolated players are equitable although players may incur different network formation costs. Our results are therefore complementary to Manea (2011b) where network formation without costs is studied. Although the implications for the bargaining outcomes are similar, the resulting network structures differ as only certain substructures, i.e. skeletons, of Manea's stable networks remain. At the same time, contrary to Manea's result, players may stay isolated in generically pairwise stable networks when network formation is costly. Moreover, nongenerically pairwise stable networks may arise resulting in heterogeneous bargaining outcomes in our model.

Our results may be extended with respect to several considerations. ${ }^{23}$ First, to model network formation, we have only used the notion of pairwise stability by Jackson and Wolinsky (1996). This approach to stability uses a very weak notion of stability as links are considered one by one. However, it is straightforward to see that generically pairwise stable networks are also robust to multiple link deletion as no player has more than two links and deleting both would leave them with zero profits. Thus, the complete characterization of generically pairwise stable networks extends to a characterization of generically pairwise Nash stable

\footnotetext{
${ }^{23}$ The extensions are discussed in more details in Gauer (2016), Sections 3.4 and 3.5.
} 
networks. Second, one may also consider the implications for welfare. Since network formation is costly and at most $\frac{n}{2}$ units of surplus can be generated, efficient networks are either the empty network or separated pairs (if $n$ is even) since the maximal amount of surplus is generated with minimal network formation cost. We can conclude that efficient networks are always generically pairwise stable in our model (if $n$ is even) and, moreover, uniquely pairwise stable if costs are large enough. Finally, abstracting from the limit case $\delta \rightarrow 1$, other networks may be generically pairwise stable. To see that, consider, for instance, very low costs $c$ and impatient players, such that $\delta$ is bounded away from 1 . In this case all links may yield a strictly positive marginal payoff since all links have a positive probability of being selected in the first stage resulting in the stability of the complete network.

Future research may consider network formation with respect to other types of market interaction. In the framework of Manea (2011a), the effect of allowing discounting may have interesting effects as pointed out above. Also, ex ante heterogeneity with respect to costs of link formation or patience may be explored. Relaxing the assumption of network stationarity would move beyond the approach of Manea (2011a) and may prove to be not as tractable as our approach (cf. e.g. Abreu and Manea, 2012a,b). More recent papers also study issues of resale and intermediation in networks (Manea, forthcoming) or bilateral trading in networks (Condorelli and Galeotti, forthcoming). Studying network formation prior to these types of market interaction may also be a worthy endeavor.

\section{Appendix}

\section{A. Proofs}

\section{A.1. Proof of Theorem 1}

Consider a component $C \subseteq N$ of some network $g$ which induces a circle or a separated pair. Then in both cases it is impossible to find a $g$-independent subset $M \subseteq C$ such that for the corresponding partner set we have $\left|L^{g}(M)\right|<|M|$. Hence, the algorithm $\mathcal{A}(g)$ yields a payoff of $\frac{1}{2}$ for each player contained in $C$ in both cases (recall Definition 1 and the subsequent payoff computation rule). Now consider two players $i, j \in N$ with $i j \notin g$ where

(a) both are contained in the same component inducing an odd circle,

(b) they are contained in different components each inducing an odd circle,

(c) they are contained in different components each inducing a separated pair,

(d) one is contained in a component inducing an odd circle and the other one is contained in a component inducing a separated pair, or

(e) one is contained in a component inducing an odd circle and the other one is an isolated player. 
Then in each of these cases the algorithm $\mathcal{A}(g+i j)$ again yields a payoff of $\frac{1}{2}$ for all players contained in the new component $C_{i}(g+i j)=C_{j}(g+i j)$. The best way to see this is again to consider Definition 1 and the subsequent payoff computation rule. ${ }^{24}$ Therefore, at least one of the two players $i$ and $j$ (in Cases (a)-(d) even both) will receive an unchanged payoff after having established this link. Regarding profits this means, however, that this player is worse off as she has to bear additional linking costs of $c>0$. Thus, the respective link will never be formed.

Next, recall the three-player case. From this it is straightforward to see that Part (i) of the theorem is indeed true. Also, we can deduce that a pairwise stable network can contain both an isolated player and a separated pair if we have linking costs $c \in\left(\frac{1}{6}, \frac{1}{2}\right]$. Together with the above Case (c) this establishes Part (ii) of the theorem.

Consider again a network $g$ and now two players $i^{\prime}, j^{\prime} \in N$ with $i^{\prime} j^{\prime} \in g$. Moreover, assume that these players are contained in a component $C$ which induces an odd circle with $m \geq 3$ players. We already know that $g$ induces a payoff of $\frac{1}{2}$ for both players. Now consider the network $g^{\prime}:=g-i^{\prime} j^{\prime}$ which is obviously a line of length $m$. Let $\left(r_{s}^{\prime}, x_{s}^{\prime}, M_{s}^{\prime}, L_{s}^{\prime}, N_{s}^{\prime}, g_{s}^{\prime}\right)_{s=1, \ldots, \bar{s}^{\prime}}$ be the outcome of $\mathcal{A}\left(g^{\prime}\right)$ (with $\left.N_{1}=C\right) .{ }^{25}$ As $m$ is an odd number, we have that $\bar{s}^{\prime}=1$ and $i^{\prime}, j^{\prime} \in M_{1}^{\prime}$. Further, it is $\left|M_{1}^{\prime}\right|=\frac{m+1}{2}$ and $\left|L_{1}^{\prime}\right|=\frac{m-1}{2}$ which implies that $v_{i^{\prime}}^{*}\left(g^{\prime}\right)=v_{j^{\prime}}^{*}\left(g^{\prime}\right)=$ $x_{1}^{\prime}=\frac{m-1}{2 m}$. As a stability condition this gives

$$
u_{i^{\prime}}^{*}(g)-u_{i^{\prime}}^{*}\left(g^{\prime}\right)=\frac{1}{2}-2 c-\left(\frac{m-1}{2 m}-c\right) \geq 0 \Leftrightarrow \frac{1}{2 m} \geq c \Leftrightarrow m \leq \frac{1}{2 c} .
$$

Of course, the same holds for player $j^{\prime}$. Together with the above Cases (a) and (b) this means that a network which is composed of odd circles is pairwise stable if and only if each circle has at most $\frac{1}{2 c}$ members. Note that a pairwise stable network can therefore contain odd circles only if we have $c \leq \frac{1}{6}$ since a circle must have at least three members by definition.

Furthermore, observe that for the cost range $c \in\left(0, \frac{1}{6}\right]$ we have $\frac{1}{2}-c>0$ which means that no player contained in a component inducing a separated pair has incentives to delete her link. This together with the above Cases (c)-(e) gives that, potentially besides one or several odd circles with a permissible number of players, a network being pairwise stable at $c \in\left(0, \frac{1}{6}\right]$ can contain separated pairs or one isolated player. As we know from the threeplayer case, however, an isolated player and a separated pair cannot coexist in a pairwise stable network at these levels of linking costs. This proves the first statement in Part (iii).

Finally, consider the transition point $c=\frac{1}{6}$. In what follows, let the network $g$ be composed of two lines of length three, an odd circle, and a separated pair as sketched in Figure A.4.

\footnotetext{
${ }^{24}$ However, a shortcut would be to consider Manea (2011a, Theorem 5) which we make use of when proving our Theorem 2.

${ }^{25}$ Disregarding players in $C^{\complement}$ is w.l.o.g. as the profile of payoffs respectively profits is componentdecomposable.
} 

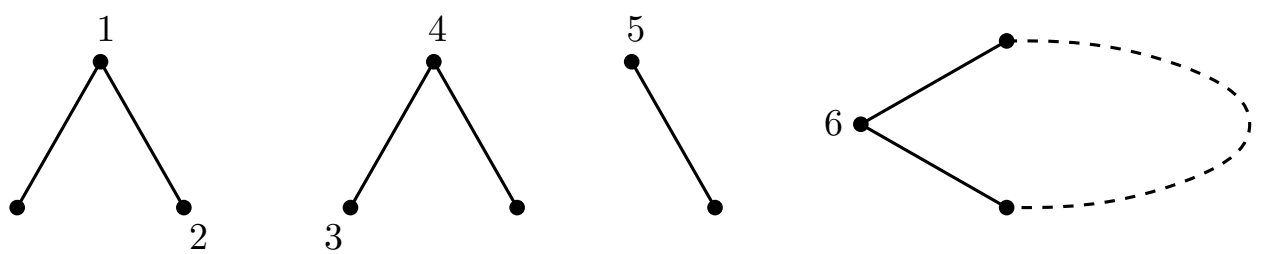

Figure A.4: A sketch of a network $g$ containing lines of length three

W.l.o.g. we focus on the labeled players $1,2, \ldots, 6$. At $c=\frac{1}{6}$ the algorithm $\mathcal{A}(g)$ yields the following profits:

$$
u_{1}^{*}(g)=\frac{2}{3}-2 c=\frac{1}{3}, \quad u_{2}^{*}(g)=u_{3}^{*}(g)=\frac{1}{3}-c=\frac{1}{6}, \quad u_{6}^{*}(g)=\frac{1}{2}-2 c=\frac{1}{6}
$$

Based on this, we are able to establish that link addition either leads to a worsening for at least one of the two players or both are indifferent. Specifically, applying the respective algorithm gives

$$
\begin{aligned}
& u_{2}^{*}(g+23)=u_{3}^{*}(g+23)=\frac{1}{2}-2 c=\frac{1}{6}=u_{2}^{*}(g)=u_{3}^{*}(g), \\
& u_{1}^{*}(g+13)=u_{1}^{*}(g+14)=u_{1}^{*}(g+15)=u_{1}^{*}(g+16)=\frac{2}{3}-3 c=\frac{1}{6}<\frac{1}{3}=u_{1}^{*}(g), \\
& u_{2}^{*}(g+25)=\frac{2}{5}-2 c=\frac{1}{15}<\frac{1}{6}=u_{2}^{*}(g), \\
& u_{6}^{*}(g+26)=\frac{1}{2}-3 c=0<\frac{1}{6}=u_{6}^{*}(g) .
\end{aligned}
$$

Since we know from the three-player case that within the component of a line of length three there are no incentives to add or delete a link at this cost level, this concludes the proof of Part (iii) and of the theorem.

\section{A.2. Proof of Proposition 1}

Consider a network $g^{\prime} \in G$ which contains disagreement links such that $g^{\prime} \backslash g^{\prime *} \neq \emptyset$ and further assume that every player has at least one link in $g^{\prime}$ implying that every player has at least one link in $g^{\prime *}$ (see Manea, 2011a, Lemma 1). ${ }^{26}$ W.l.o.g. let $12 \in g^{\prime} \backslash g^{\prime *}$ and denote $g:=g^{\prime}-12$ implying $g^{\prime *} \subseteq g$. For both networks consider the outcome of the algorithms $\mathcal{A}(g)$ denoted by $\left(r_{s}, x_{s}, M_{s}, L_{s}, N_{s}, g_{s}\right)_{s=1, \ldots, \bar{s}}$ and $\mathcal{A}\left(g^{\prime}\right)$ denoted by $\left(r_{s}^{\prime}, x_{s}^{\prime}, M_{s}^{\prime}, L_{s}^{\prime}, N_{s}^{\prime}, g_{s}^{\prime}\right)_{s=1, \ldots, \bar{s}^{\prime}}$. We first note the following properties of $g, g^{\prime}$ and $g^{\prime *}$.

P.1 If $i j \in g$, then $v_{i}^{*}\left(g^{\prime}\right)+v_{j}^{*}\left(g^{\prime}\right) \geq 1$, and if $i j \in g^{\prime *}$, then $v_{i}^{*}\left(g^{\prime *}\right)+v_{j}^{*}\left(g^{\prime *}\right)=1$ (since $i j \in g \Rightarrow i j \in g^{\prime}$, compare to Manea, 2011a, Proposition 2).

P.2 For all $g^{\prime *}$-independent sets $M$ the equilibrium payoffs satisfy (by Manea, 2011a, Theorem 3$)$ :

$$
\min _{i \in M} v_{i}^{*}\left(g^{\prime}\right) \leq \frac{\left|L^{g^{\prime *}}(M)\right|}{|M|+\left|L^{g^{\prime *}}(M)\right|}, \quad \max _{j \in L^{g^{\prime *}}(M)} v_{j}^{*}\left(g^{\prime}\right) \geq \frac{|M|}{|M|+\left|L^{g^{\prime *}}(M)\right|}
$$

\footnotetext{
${ }^{26}$ This is w.l.og. since we can disregard isolated players who do not affect payoffs of other players by component decomposability.
} 
We show that $\mathcal{A}\left(g^{\prime}\right)$ yields the same outcome as $\mathcal{A}(g)$. To see this, we can simply follow the proof steps for the payoff computation rule in Theorem 4 in Manea (2011a). Let $s<\bar{s}$ and suppose we have shown that the outcomes of $\mathcal{A}(g)$ and $\mathcal{A}\left(g^{\prime}\right)$ are identical up to $s-1 .^{27}$

Let $\underline{x}_{s}^{\prime}:=\min _{i \in N_{s}} v_{i}^{*}\left(g^{\prime}\right)$. Completely analogously to the proof of Claim 4.1 in Manea (2011a), we can first show that $\underline{x}_{s}^{\prime} \leq x_{s}$ by contradiction. Supposing $\underline{x}_{s}^{\prime}>x_{s}$, we get by Proposition 3 in Manea (2011a), induction hypothesis, and P.1 that $L^{g^{\prime *}}\left(M_{s}\right) \subseteq L^{g_{s}}\left(M_{s}\right)=$ $L_{s}$. Since $M_{s}$ is $g$-independent and $g^{\prime *} \subseteq g, M_{s}$ is also $g^{\prime *}$-independent, and, hence, applying P.2 we receive $\underline{x}_{s}^{\prime}=\min _{i \in N_{s}} v_{i}^{*}\left(g^{\prime}\right) \leq \min _{i \in M_{s}} v_{i}^{*}\left(g^{\prime}\right) \leq \frac{\left|L^{g^{\prime *}}\left(M_{s}\right)\right|}{\left|M_{s}\right|+\left|L^{g^{\prime *}}\left(M_{s}\right)\right|} \leq \frac{\left|L_{s}\right|}{\left|M_{s}\right|+\left|L_{s}\right|}=x_{s}$, contradicting $x_{s}<\underline{x}_{s}^{\prime}$.

Thus, we have $r_{s}^{\prime}<1$ and, hence $s<\bar{s}^{\prime}$. We then get from Theorem 4 in Manea (2011a) that $x_{s}^{\prime}=\underline{x}_{s}^{\prime}, M_{s}^{\prime}=\arg \min _{i \in N_{s}} v_{i}^{*}\left(g^{\prime}\right)$ and $M_{s}^{\prime}$ is $g_{s}^{\prime}$-independent and $L_{s}^{\prime}=L^{g_{s}^{\prime}}\left(M_{s}^{\prime}\right)$. Since $g_{s} \subseteq g_{s}^{\prime}$ implying $L_{s}^{\prime}=L^{g_{s}^{\prime}}\left(M_{s}^{\prime}\right) \supseteq L^{g_{s}}\left(M_{s}^{\prime}\right)$, we get $x_{s}^{\prime}=\frac{\left|L_{s}^{\prime}\right|}{\left|L_{s}^{\prime}\right|+\left|M_{s}^{\prime}\right|} \geq \frac{\left|L^{g_{s}}\left(M_{s}^{\prime}\right)\right|}{\left|L^{g_{s}}\left(M_{s}^{\prime}\right)\right|+\left|M_{s}^{\prime}\right|}$ and, thus, $\frac{x_{s}^{\prime}}{1-x_{s}^{\prime}} \geq \frac{\left|L^{g_{s}}\left(M_{s}^{\prime}\right)\right|}{\left|M_{s}^{\prime}\right|}$. But, $r_{s}$ minimizes the shortage ratio in $g_{s}$ and $M_{s}^{\prime}$ is also $g_{s}$-independent since $g_{s} \subseteq g_{s}^{\prime}$. Therefore, $\frac{x_{s}}{1-x_{s}}=r_{s} \leq \frac{\left|L^{g_{s}}\left(M_{s}^{\prime}\right)\right|}{\left|M_{s}^{\prime}\right|}$ and hence, $x_{s} \leq x_{s}^{\prime}$.

We conclude, $x_{s}=x_{s}^{\prime}$ and thereby $r_{s}=\frac{\left|L^{g_{s}}\left(M_{s}\right)\right|}{\left|M_{s}\right|}=\frac{\left|L^{g_{s}}\left(M_{s}^{\prime}\right)\right|}{\left|M_{s}^{\prime}\right|}=\frac{\left|L^{g_{s}^{\prime}}\left(M_{s}^{\prime}\right)\right|}{\left|M_{s}^{\prime}\right|}=r_{s}^{\prime}$. Further, as $L^{g_{s}}\left(M_{s}^{\prime}\right) \subseteq L^{g_{s}^{\prime}}\left(M_{s}^{\prime}\right)=L_{s}^{\prime}$, and $\frac{\left|L^{g_{s}}\left(M_{s}^{\prime}\right)\right|}{\left|M_{s}^{\prime}\right|}=\frac{\left|L_{s}^{g_{s}^{\prime}}\left(M_{s}^{\prime}\right)\right|}{\left|M_{s}^{\prime}\right|}$, we must have $L^{g_{s}}\left(M_{s}^{\prime}\right)=L_{s}^{\prime}$.

Since $M_{s}^{\prime}$ is $g_{s}$-independent and $M_{s}$ is the union of all $g_{s}$-independent sets minimizing the shortage ratio in $g_{s}$, we get $M_{s}^{\prime} \subseteq M_{s}$. To show that $M_{s}^{\prime}=M_{s}$, we follow the reasoning of the proof of Claim 4.4 in Manea (2011a). To get a contradiction, assume $M_{s} \backslash M_{s}^{\prime} \neq \emptyset$. Applying Lemma 4 in Manea (2011a) to $g$ and P.1 to $g^{\prime *}$, we can conclude analogously that $\left|L^{g^{\prime *}}\left(M_{s} \backslash M_{s}^{\prime}\right)\right| \leq\left|L_{s} \backslash L_{s}^{\prime}\right|$. Since $L_{s}^{\prime}=L^{g_{s}^{\prime}}\left(M_{s}^{\prime}\right)=L^{g_{s}}\left(M_{s}^{\prime}\right) \subseteq L^{g_{s}}\left(M_{s}\right)=L_{s}$, we get $\left|L_{s} \backslash L_{s}^{\prime}\right|=\left|L_{s}\right|-\left|L_{s}^{\prime}\right|=r_{s}\left(\left|M_{s}\right|-\left|M_{s}^{\prime}\right|\right)$, where the last equality is due to $r_{s}=\frac{\left|L_{s}\right|}{\left|M_{s}\right|}=$

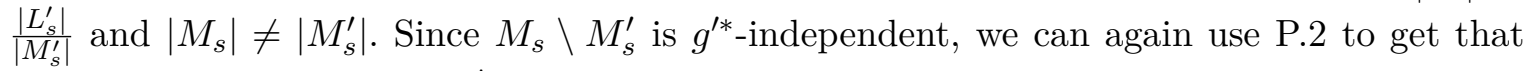
$\min _{i \in M_{s} \backslash M_{s}^{\prime}} v_{i}^{*}\left(g^{\prime}\right) \leq \frac{\left|L^{g^{\prime *}}\left(M_{s} \backslash M_{s}^{\prime}\right)\right|}{\left|M_{s} \backslash M_{s}^{\prime}\right|+\left|L^{g^{\prime *}}\left(M_{s} \backslash M_{s}^{\prime}\right)\right|} \leq \frac{\left|L_{s}\right|-\left|L_{s}^{\prime}\right|}{\left|M_{s}\right|-\left|M_{s}^{\prime}\right|+\left|L_{s}\right|-\left|L_{s}^{\prime}\right|}=\frac{r_{s}}{1+r_{s}}=x_{s}$, contradicting $v_{i}^{*}\left(g^{\prime}\right)>x_{s}$ for all $i \in N_{s} \backslash M_{s}^{\prime}$. Thus, $\mathcal{A}(g)$ and $\mathcal{A}\left(g^{\prime}\right)$ yield the same outcome for all $s<\bar{s}$.

Finally, $v_{k}^{*}\left(g^{\prime}\right)=\frac{1}{2}$ for all $k \in N_{\bar{s}}$ (if nonempty). This is because $v_{k}^{*}(g)=\frac{1}{2}$ for all $k \in N_{\bar{s}}$ and $g_{\bar{s}} \subseteq g_{\bar{s}}^{\prime}$ implying that for any $g_{\bar{s}}^{\prime}$-independent set $M, 1 \leq \frac{\left|L^{g_{\bar{s}}}(M)\right|}{|M|} \leq \frac{\left|L^{g_{\bar{s}}^{\prime}}(M)\right|}{|M|}$.

Altogether we get that $v_{i}^{*}(g)=v_{i}^{*}\left(g^{\prime}\right)$ for all $i \in N$. Since 12 is costly, we therefore get $u_{1}\left(g^{\prime}\right)-u_{1}(g)=u_{2}\left(g^{\prime}\right)-u_{2}(g)=-c$ implying that $g^{\prime}$ cannot be pairwise stable. Thus, for any pairwise stable $g$, we get $g=g^{*}$, and, hence, $v_{i}^{*}(g)+v_{j}^{*}(g)=1$ for all $i j \in g$ by Manea (2011a), Proposition 2.

\section{A.3. Proof of Theorem 2}

Consider a pairwise stable network $g$ and assume that $\left.g\right|_{\tilde{N}(g)}$ is not a union of separated pairs and odd circles. Notice that due to Proposition 1 for any component $C \subseteq N$ of $g$ it must either be $C \subseteq \tilde{N}(g)$ or $C \subseteq \tilde{N}(g)^{\complement}$. Furthermore, recall that the profile of payoffs

\footnotetext{
${ }^{27}$ For $s=1$ all the assumptions used in the inductive step are satisfied, see Manea (2011a), Footnote 17.
} 
is component-decomposable, meaning that $v_{i}^{*}(g)=v_{i}^{*}\left(\left.g\right|_{\tilde{N}(g)}\right)$ for all $i \in \tilde{N}(g)$. Thus, the network $\left.g\right|_{\tilde{N}(g)}$ is equitable such that by Manea (2011a, Theorem 5) respectively by Berge (1981) it has a so called edge cover composed of separated pairs and odd circles. This means that there exists a union of separated pairs and odd circles $\left.g^{\prime} \subseteq g\right|_{\tilde{N}(g)}$ such that no player $i \in \tilde{N}(g)$ is isolated in $g^{\prime}$. By assumption there must exist a link $\left.i j \in g\right|_{\tilde{N}(g)} \backslash g^{\prime}$. Obviously, the network $g^{\prime}$ is also an edge cover of the network $\left.g\right|_{\tilde{N}(g)}-i j$. Again from Manea (2011a, Theorem 5) respectively from Berge (1981) it then follows that $\left.g\right|_{\tilde{N}(g)}-i j$ is still equitable. Hence, recalling the implication of Proposition 1 mentioned above, this gives

$$
\begin{aligned}
u_{i}^{*}(g)=v_{i}^{*}\left(\left.g\right|_{\tilde{N}(g)}\right)-\eta_{i}\left(\left.g\right|_{\tilde{N}(g)}\right) c=\frac{1}{2}-\eta_{i}\left(\left.g\right|_{\tilde{N}(g)}\right) & <\frac{1}{2}-\left(\eta_{i}\left(\left.g\right|_{\tilde{N}(g)}\right)-1\right) c \\
& =v_{i}^{*}\left(\left.g\right|_{\tilde{N}(g)}-i j\right)-\eta_{i}\left(\left.g\right|_{\tilde{N}(g)}-i j\right) c \\
& =u_{i}^{*}(g-i j) .
\end{aligned}
$$

Thus, arriving at a contradiction, this concludes the proof.

\section{A.4. Proof of Lemma 1 and Lemma 2}

As announced in Section 3, the proofs of both lemmas rest on another rather technical lemma which be provide and prove first.

Lemma 3. Let $\tilde{g}$ be a network with $\mathcal{A}(\tilde{g})$ providing $\left(\tilde{r}_{s}, \tilde{x}_{s}, \tilde{M}_{s}, \tilde{L}_{s}, \tilde{N}_{s}, \tilde{g}_{s}\right)_{s}$. For any step $s<\bar{s}$ and any set $I \subseteq N$ the following implications must apply:

$$
\begin{array}{lll}
\text { (i) } 1 \leq\left|\tilde{M}_{s} \cap I\right| \leq\left|\tilde{L}_{s} \cap I\right| & \Rightarrow & \left|L^{\tilde{g}_{s}}\left(\tilde{L}_{s} \cap I\right) \cap \tilde{M}_{s} \cap I^{\complement}\right| \geq 1, \\
\text { (ii) } 1 \leq\left|\tilde{M}_{s} \cap I\right|<\left|\tilde{L}_{s} \cap I\right| & \Rightarrow & \left|L^{\tilde{g}_{s}}\left(\tilde{L}_{s} \cap I\right) \cap \tilde{M}_{s} \cap I^{\complement}\right| \geq 2 .
\end{array}
$$

Proof of Lemma 3. We prove the two parts of the lemma one after the other.

Part (i):

Assume that we have $1 \leq\left|\tilde{M}_{s} \cap I\right| \leq\left|\tilde{L}_{s} \cap I\right|$ and $L^{\tilde{g}_{s}}\left(\tilde{L}_{s} \cap I\right) \cap \tilde{M}_{s} \cap I^{\complement}=\emptyset$ for some step $s<\bar{s}$ and some set $I \subseteq N$. Recalling Definition 1, this obviously implies

$$
\frac{\left|\tilde{L}_{s}\right|}{\left|\tilde{M}_{s}\right|}=\tilde{r}_{s}<1 \leq \frac{\left|\tilde{L}_{s} \cap I\right|}{\left|\tilde{M}_{s} \cap I\right|} .
$$

Additionally, we have that $\tilde{M}_{s}=\left(\tilde{M}_{s} \cap I\right) \dot{\cup}\left(\tilde{M}_{s} \backslash I\right)$ and $\tilde{L}_{s}=\left(\tilde{L}_{s} \cap I\right) \dot{\cup}\left(\tilde{L}_{s} \backslash I\right)$. This induces that $\tilde{M}_{s} \backslash I \neq \emptyset$ since it is $\left|\tilde{M}_{s} \cap I\right| \leq\left|\tilde{L}_{s} \cap I\right| \leq\left|\tilde{L}_{s}\right|$ but $\left|\tilde{M}_{s}\right|>\left|\tilde{L}_{s}\right|$. It follows that

$$
\frac{\left|\tilde{L}_{s} \backslash I\right|}{\left|\tilde{M}_{s} \backslash I\right|}<\frac{\left|\tilde{L}_{s}\right|}{\left|\tilde{M}_{s}\right|} .
$$

Moreover, it is $L^{\tilde{g}_{s}}\left(\tilde{M}_{s} \backslash I\right) \subseteq \tilde{L}_{s} \backslash I$ since by assumption $L^{\tilde{g}_{s}}\left(\tilde{L}_{s} \cap I\right) \cap \tilde{M}_{s} \subseteq I$. Taken together, this then gives

$$
\frac{\left|L^{\tilde{g}_{s}}\left(\tilde{M}_{s} \backslash I\right)\right|}{\left|\tilde{M}_{s} \backslash I\right|} \leq \frac{\left|\tilde{L}_{s} \backslash I\right|}{\left|\tilde{M}_{s} \backslash I\right|}<\frac{\left|\tilde{L}_{s}\right|}{\left|\tilde{M}_{s}\right|}=\tilde{r}_{s}
$$


which contradicts the minimality of $\tilde{r}_{s}$.

Part (ii):

It remains to show that having $1 \leq\left|\tilde{M}_{s} \cap I\right|<\left|\tilde{L}_{s} \cap I\right|$ and $\left|L^{\tilde{g}_{s}}\left(\tilde{L}_{s} \cap I\right) \cap \tilde{M}_{s} \cap I^{\complement}\right|=1$ in some step $s<\bar{s}$ and for some set $I \subseteq N$ leads to a contradiction as well. In such a situation, slightly different from Part (i), we have

$$
\frac{\left|\tilde{L}_{s}\right|}{\left|\tilde{M}_{s}\right|}=\tilde{r}_{s}<1 \leq \frac{\left|\tilde{L}_{s} \cap I\right|}{\left|\tilde{M}_{s} \cap I\right|+1} .
$$

Again, it holds that $\tilde{M}_{s}=\left(\tilde{M}_{s} \cap I\right) \dot{\cup}\left(\tilde{M}_{s} \backslash I\right)$ and $\tilde{L}_{s}=\left(\tilde{L}_{s} \cap I\right) \dot{\cup}\left(\tilde{L}_{s} \backslash I\right)$, which in this case even guarantees that $\left|\tilde{M}_{s} \backslash I\right| \geq 2$ since it is $\left|\tilde{M}_{s} \cap I\right|<\left|\tilde{L}_{s} \cap I\right| \leq\left|\tilde{L}_{s}\right|$, but $\left|\tilde{M}_{s}\right|>\left|\tilde{L}_{s}\right|$. This gives

$$
\frac{\left|\tilde{L}_{s} \backslash I\right|}{\left|\tilde{M}_{s} \backslash I\right|-1}<\frac{\left|\tilde{L}_{s}\right|}{\left|\tilde{M}_{s}\right|}
$$

Moreover, we have that there exists exactly one player $\tilde{i} \in L^{\tilde{g}_{s}}\left(\tilde{L}_{s} \cap I\right) \cap \tilde{M}_{s} \cap I^{\complement}$. Similarly to Part (i) this implies that it is $L^{\tilde{g}_{s}}\left(\tilde{M}_{s} \backslash(I \dot{\cup}\{\tilde{i}\})\right) \subseteq \tilde{L}_{s} \backslash I$, which combined with the above leads to

$$
\frac{\left|L^{\tilde{g}_{s}}\left(\tilde{M}_{s} \backslash(I \dot{\cup}\{\tilde{i}\})\right)\right|}{\left|\tilde{M}_{s} \backslash(I \dot{\cup}\{\tilde{i}\})\right|} \leq \frac{\left|\tilde{L}_{s} \backslash I\right|}{\left|\tilde{M}_{s} \backslash I\right|-1}<\frac{\left|\tilde{L}_{s}\right|}{\left|\tilde{M}_{s}\right|}=\tilde{r}_{s},
$$

which again contradicts the minimality of $\tilde{r}_{s}$.

Now, we can turn to the proof of the first of the two lemmas which are stated in Section 3.

Proof of Lemma 1. For $i, j \in M_{1}$ consider the network $g^{\prime}:=g+i j$. Let $\left(r_{s}^{\prime}, x_{s}^{\prime}, M_{s}^{\prime}, L_{s}^{\prime}, N_{s}^{\prime}, g_{s}^{\prime}\right)_{s=1, \ldots, \bar{s}^{\prime}}$ be the outcome of $\mathcal{A}\left(g^{\prime}\right)$. Assume for contradiction that there exists a step $\hat{s} \in\left\{1, \ldots, \bar{s}^{\prime}-1\right\}$ such that $L_{1} \cap M_{s}^{\prime}=M_{1} \cap L_{s}^{\prime}=\emptyset$ for all $s \in\{1, \ldots, \hat{s}-1\}$ but $M_{1} \cap L_{\hat{s}}^{\prime} \neq \emptyset$. Note that $L_{1} \cap M_{\hat{s}}^{\prime} \neq \emptyset$ would also entail $M_{1} \cap L_{\hat{s}}^{\prime} \neq \emptyset$. This is because any player contained in $L_{1} \cap M_{\hat{s}}^{\prime}$ must have a neighbor $k \in M_{1}$ in $g$ due to the minimality of $r_{1}<1$ and it can obviously neither be $k \in L_{s}^{\prime}$ nor $k \in M_{s}^{\prime}$ for any $s \in\{1, \ldots, \hat{s}-1\}$. In what follows, we construct a sequence of players $\left(i_{0}, i_{1}, i_{2}, \ldots\right)$ and show by induction that the underlying procedure which sequentially adds players to it can never break up so that we get a contradiction to the finiteness of the player set $N$. For $m \in \mathbb{N}$ let $I_{m}:=\left\{i_{0}, i_{1}, \ldots, i_{m}\right\} \subseteq N$ denote the players of the sequence up to the $m$ th one. We need to distinguish two cases.

Case 1: $i \in L_{\hat{s}}^{\prime}$

Set $i_{0}=i$. It then must be $\left|N_{i_{0}}\left(g_{\hat{s}}^{\prime}\right) \cap M_{\hat{s}}^{\prime}\right| \geq 2$ since otherwise one could reduce $r_{\hat{s}}^{\prime}$ by not including $i_{0}$ and possibly her one contact belonging to $M_{\hat{s}}^{\prime}$. This guarantees that there exists $i_{1} \in N_{i_{0}}\left(g_{\hat{s}}^{\prime}\right) \cap M_{\hat{s}}^{\prime} \backslash\{j\}$. So it is $i_{0} \in M_{1} \cap L_{\hat{s}}^{\prime}$ and $i_{1} \in L_{1} \cap M_{\hat{s}}^{\prime}$. Let $I_{1}=\left\{i_{0}, i_{1}\right\}$. Now consider some odd number $m \in \mathbb{N}$. Assume that $L_{1} \cap I_{m}=M_{\hat{s}}^{\prime} \cap I_{m}, M_{1} \cap I_{m}=L_{\hat{s}}^{\prime} \cap I_{m}$ and 
that the cardinalities of these two sets are equal. We then have:

- It is $1 \leq\left|M_{1} \cap I_{m}\right|=\left|L_{1} \cap I_{m}\right|$ and therefore by Lemma 3(i) there exists a player $i_{m+1} \in L^{g}\left(L_{1} \cap I_{m}\right) \cap M_{1} \cap I_{m}^{\complement}$. For this player it must hold that $i_{m+1} \in M_{1} \cap L_{\hat{s}}^{\prime} \backslash I_{m}$ since $L_{1} \cap I_{m} \subseteq M_{\hat{s}}^{\prime}$ and $M_{1} \cap L_{s}^{\prime}=\emptyset$ for all $s \in\{1, \ldots, \hat{s}-1\}$.

- It then is $1 \leq\left|M_{\hat{s}}^{\prime} \cap I_{m+1}\right|<\left|L_{\hat{s}}^{\prime} \cap I_{m+1}\right|$ and therefore by Lemma 3(ii) there exists a player $i_{m+2} \in L^{g_{\hat{s}}^{\prime}}\left(L_{\hat{s}}^{\prime} \cap I_{m+1}\right) \cap M_{\hat{s}}^{\prime} \cap I_{m+1}^{\complement} \backslash\{j\}$. For this player it must hold that $i_{m+2} \in L_{1} \cap M_{\hat{s}}^{\prime} \backslash I_{m+1}$ since $L_{\hat{s}}^{\prime} \cap I_{m+1} \subseteq M_{1}$ and $i_{m+2} \neq j$.

Thus, it is $L_{1} \cap I_{m+2}=M_{\hat{s}}^{\prime} \cap I_{m+2}, M_{1} \cap I_{m+2}=L_{\hat{s}}^{\prime} \cap I_{m+2}$ and also the cardinalities of these two sets are equal. Moreover, it is $\left|I_{m+2}\right|=\left|I_{m}\right|+2$. By induction it follows that the player set $N$ must be infinitely large. Thus, we arrive at a contradiction.

Case 2: $i \notin L_{\hat{s}}^{\prime}$

In this case we must have $j \notin M_{\hat{s}}^{\prime}$ since by assumption $M_{1} \cap L_{s}^{\prime}=\emptyset$ for all $s \in\{1, \ldots, \hat{s}-1\}$. For the same reason, $i \in M_{\hat{s}}^{\prime}$ would imply $j \in L_{\hat{s}}^{\prime}$ which is equivalent to Case 1 . This is also true for $i \notin M_{\hat{s}}^{\prime}$ and $j \in L_{\hat{s}}^{\prime}$. So it remains to consider the case that $i, j \notin\left(M_{\hat{s}}^{\prime} \cup L_{\hat{s}}^{\prime}\right)$. However, by assumption there must be a player $i_{0} \in M_{1} \cap L_{\hat{s}}^{\prime}$. As in the previous case, existence of another player $i_{1} \in N_{i_{0}}\left(g_{\hat{s}}^{\prime}\right) \cap M_{\hat{s}}^{\prime}$ is then guaranteed and it must be $i_{1} \notin\{i, j\}$ since $i, j \notin M_{\hat{s}}^{\prime}$. Therefore it is $i_{1} \in L_{1} \cap M_{\hat{s}}^{\prime}$. Let again $I_{1}=\left\{i_{0}, i_{1}\right\}$ and assume for some odd number $m \in \mathbb{N}$ that $L_{1} \cap I_{m}=M_{\hat{s}}^{\prime} \cap I_{m}, M_{1} \cap I_{m}=L_{\hat{s}}^{\prime} \cap I_{m}$ and that the cardinalities of these two sets are equal. Furthermore, assume that $i, j \notin I_{m}$. Similarly to the first case we have:

- There exists $i_{m+1} \in M_{1} \cap L_{\hat{s}}^{\prime} \backslash I_{m}$ for the stated reasons.

- By Lemma 3(ii) there then exists a player $i_{m+2} \in L^{g_{\hat{s}}^{\prime}}\left(L_{\hat{s}}^{\prime} \cap I_{m+1}\right) \cap M_{\hat{s}}^{\prime} \cap I_{m+1}^{\complement}$. For this player it must hold that $i_{m+2} \in L_{1} \cap M_{\hat{s}}^{\prime} \backslash I_{m+1}$ since $L_{\hat{s}}^{\prime} \cap I_{m+1} \subseteq M_{1} \backslash\{i, j\}$.

Thus, it is again $L_{1} \cap I_{m+2}=M_{\hat{s}}^{\prime} \cap I_{m+2}, M_{1} \cap I_{m+2}=L_{\hat{s}}^{\prime} \cap I_{m+2}$ and also the cardinalities of these two sets are equal. Beyond that, we have $i, j \notin I_{m+2}$. By induction this leads again to a contradiction to the finiteness of the player set $N$.

Summing up, we have that $L_{1} \cap M_{s}^{\prime}=M_{1} \cap L_{s}^{\prime}=\emptyset$ for all $s<\bar{s}^{\prime}$. Therefore, it must be $v_{i}^{*}\left(g^{\prime}\right), v_{j}^{*}\left(g^{\prime}\right) \leq \frac{1}{2}$. On the contrary, we know by Manea (2011a, Proposition 2) that $v_{i}^{*}\left(g^{\prime}\right)+v_{j}^{*}\left(g^{\prime}\right) \geq 1$. Taken together, this implies $v_{i}^{*}\left(g^{\prime}\right)=v_{j}^{*}\left(g^{\prime}\right)=\frac{1}{2}$.

With regard to the second part of the lemma consider the network $g^{\prime}:=g+i(n+1)$ and let $\left(r_{s}^{\prime}, x_{s}^{\prime}, M_{s}^{\prime}, L_{s}^{\prime}, N_{s}^{\prime}, g_{s}^{\prime}\right)_{s=1, \ldots, \bar{s}^{\prime}}$ be the outcome of $\mathcal{A}\left(g^{\prime}\right)$. It is clear that $n+1 \notin L_{s}^{\prime}$ for all $s<\bar{s}^{\prime}$ since otherwise one could simply reduce $r_{s}^{\prime}$ by deleting $n+1$ from $L_{s}^{\prime}$ and possibly her one neighbor $i$ from $M_{s}^{\prime}$. The possibility that $i \in L_{s}^{\prime}$ for some $s<\bar{s}^{\prime}$ can be ruled out by a line of argumentation which is equivalent to the proof of the first part if one substitutes $n+1$ for $j, M_{2}$ for $M_{1}$ and $L_{2}$ for $L_{1}$ (while taking into account that $\mathcal{A}(g)$ provides $M_{1}=\{n+1\}$ and $L_{1}=\emptyset$ in this case). 
And finally we establish the second of the two lemmas.

Proof of Lemma 2. W.l.o.g. assume that $g$ has only one component. ${ }^{28}$ Beside $g$ consider the network $g^{\prime}:=g-k l$ and let $\left(r_{s}^{\prime}, x_{s}^{\prime}, M_{s}^{\prime}, L_{s}^{\prime}, N_{s}^{\prime}, g_{s}^{\prime}\right)_{s^{\prime}=1, \ldots, \bar{s}^{\prime}}$ be the outcome of $\mathcal{A}\left(g^{\prime}\right)$. Similarly to the proof of Lemma 1 assume for contradiction that there exists a step $\hat{s} \in\left\{1, \ldots, \bar{s}^{\prime}-1\right\}$ such that $L_{1} \cap M_{s}^{\prime}=M_{1} \cap L_{s}^{\prime}=\emptyset$ for all $s \in\{1, \ldots, \hat{s}-1\}$ but $L_{1} \cap M_{\hat{s}}^{\prime} \neq \emptyset$. Observe that $M_{1} \cap L_{\hat{s}}^{\prime} \neq \emptyset$ would also entail $L_{1} \cap M_{\hat{s}}^{\prime} \neq \emptyset$ since due to the minimality of $r_{\hat{s}}^{\prime}$ any player in $M_{1} \cap L_{\hat{s}}^{\prime}$ needs to have a $g^{\prime}$-neighbor in $M_{\hat{s}}^{\prime}$ who then must have been a neighbor in $g$ as well. We again construct a sequence of players $\left(i_{0}, i_{1}, i_{2}, \ldots\right)$ and show by induction that the underlying procedure which sequentially adds players to it can never break up, meaning that we get a contradiction to the finiteness of the player set $N$. For $m \in \mathbb{N}$ let $I_{m}:=\left\{i_{0}, i_{1}, \ldots, i_{m}\right\} \subseteq N$ denote the players of the sequence up to the $m$ th one.

Initially, select some player $i_{0} \in L_{1} \cap M_{\hat{s}}^{\prime}$. $i_{0}$ cannot be isolated or a loose-end player, i.e. she must have more than one link in $g$, since otherwise one could reduce $r_{1}$ by not including $i_{0}$ in $L_{1}$ and possibly her one contact in $M_{1}$. This guarantees that there exists $i_{1} \in N_{i_{0}}\left(g^{\prime}\right)$. It must be $i_{1} \in M_{1} \cap L_{\hat{s}}^{\prime}$ since by assumption $M_{1} \cap L_{s}^{\prime}=\emptyset$ for all $s \in\{1, \ldots, \hat{s}-1\}$. Let $I_{1}=\left\{i_{0}, i_{1}\right\}$. Now consider some odd number $m \in \mathbb{N}$. Assume that $L_{1} \cap I_{m}=M_{\hat{s}}^{\prime} \cap I_{m}$, $M_{1} \cap I_{m}=L_{\hat{s}}^{\prime} \cap I_{m}$ and that the cardinalities of these two sets are equal. We then have:

- It is $1 \leq\left|M_{\hat{s}}^{\prime} \cap I_{m}\right|=\left|L_{\hat{s}}^{\prime} \cap I_{m}\right|$ and therefore by Lemma 3(i) there exists a player $i_{m+1} \in L^{g_{\hat{s}}^{\prime}}\left(L_{\hat{s}}^{\prime} \cap I_{m}\right) \cap M_{\hat{s}}^{\prime} \cap I_{m}^{\complement}$. For this player it must hold that $i_{m+1} \in L_{1} \cap M_{\hat{s}}^{\prime} \backslash I_{m}$ since it is $L_{\hat{s}}^{\prime} \cap I_{m} \subseteq M_{1}$.

- Then it is $1 \leq\left|M_{1} \cap I_{m+1}\right|<\left|L_{1} \cap I_{m+1}\right|$ and therefore by Lemma 3(ii) there exists a player $i_{m+2} \in L^{g}\left(L_{1} \cap I_{m+1}\right) \cap M_{1} \cap I_{m+1}^{\complement} \cap L^{g_{\hat{s}}^{\prime}}\left(L_{1} \cap I_{m+1}\right)$ since $g^{\prime}$ arose from $g$ by a single link deletion and, additionally, $M_{1} \cap L_{s}^{\prime}=\emptyset$ for all $s \in\{1, \ldots, \hat{s}-1\}$ and $L_{1} \cap I_{m+1} \subseteq M_{\hat{s}}^{\prime}$. This reasoning then also implies that $i_{m+2} \in M_{1} \cap L_{\hat{s}}^{\prime} \backslash I_{m+1}$.

Thus it is $L_{1} \cap I_{m+2}=M_{\hat{s}}^{\prime} \cap I_{m+2}, M_{1} \cap I_{m+2}=L_{\hat{s}}^{\prime} \cap I_{m+2}$ and also the cardinalities of these two sets are equal. Moreover, it is $\left|I_{m+2}\right|=\left|I_{m}\right|+2$. Again, by induction this leads to a contradiction to the finiteness of the player set $N$. Consequently, it must be $L_{1} \cap M_{s}^{\prime}=M_{1} \cap L_{s}^{\prime}=\emptyset$ for all $s<\bar{s}^{\prime}$. Since $L_{1}$ and $M_{s}$ do not intersect for any $s \in \mathbb{N}$, we get $v_{j}^{*}(g-k l) \geq \frac{1}{2}$ for all $j \in L_{1}$.

\section{A.5. Proof of Proposition 2}

First note that by Theorems 1-3 any nongenerically pairwise stable $\hat{g}$ contains at least one nonequitable component $C$ of at least three players for $c<\frac{1}{2}$. In what follows the Proposition's four parts are shown separately. By component decomposability, we restrict

\footnotetext{
${ }^{28}$ Again, this is w.l.o.g. as the profile of payoffs respectively profits is component-decomposable.
} 
w.l.o.g. to nongenercially pairwise stable networks that consist only of one such nonequitable component $C$, i.e. where $g:=\left.\hat{g}\right|_{C}$.

Proof of Proposition 2(i). Let $g$ be a tree with $n>3$ players and assume that $g$ is pairwise stable. By Theorem 2 it cannot be the case that all players receive a payoff of $\frac{1}{2}$ in $g$. According to Proposition 1 and Corollary 2, the algorithm $\mathcal{A}(g)$ therefore has to stop after the first step providing an outcome $\left(r_{1}, x_{1}, M_{1}, L_{1}, N_{1}, g_{1}\right)$ with $M_{1} \dot{\cup} L_{1}=N,\left|M_{1}\right|>\left|L_{1}\right|$ and $\left.g\right|_{M_{1}}=\left.g\right|_{L_{1}}=\emptyset$. So we have $r_{1} \in(0,1)$ and $v_{i}^{*}(g)=1-v_{j}^{*}(g)=x_{1} \in\left(0, \frac{1}{2}\right)$ for all $i \in M_{1}, j \in L_{1}$. Theorem 3 then implies that

$$
x_{1}+c=\frac{1}{2} .
$$

The class of tree networks we consider here can be divided into the following subclasses:

(a) No player has more than two links in $g$, meaning that $g$ is a line network.

(b) There is a player who has more than two links in $g$ such that at least two of her neighbors are loose-end players. ${ }^{29}$

(c) There is a player who has more than two links in $g$ but no player has more than one loose-end contact.

In the following, we distinguish between these three subclasses and show separately that there arises a contradiction to pairwise stability.

Subclass (a):

W.l.o.g. let $g:=\{12,23, \ldots,(n-1) n\}$. Here $n$ must be odd since otherwise it would obviously be $\frac{\left|L^{g}(M)\right|}{|M|} \geq 1$ for all $g$-independent sets $M \subseteq N$ inducing a payoff of $\frac{1}{2}$ for every player. So by assumption it must be $n \geq 5$. Considering the algorithm $\mathcal{A}(g)$, we find that the shortage ratio is minimized by the $g$-independent set which contains the players $1,3, \ldots, n-2, n$. Therefore, it is $r_{1}=\frac{n-1}{n+1}$ and $x=\frac{n-1}{2 n}$. Hence, by (A.1),

$$
c=\frac{1}{2 n} \text {. }
$$

Now, if player 3 deletes her link to player 2, then she becomes a loose-end player. Moreover, in the network $g-23$ she is contained in a component with an odd number of players which induces a line of length $n-2$. Hence, it is $v_{3}^{*}(g-23)=\frac{n-3}{2(n-2)}$. Taking into account equation (A.2), the corresponding stability condition yields

$$
\begin{aligned}
u_{3}^{*}(g)-u_{3}^{*}(g-23) \geq 0 & \Leftrightarrow \quad v_{3}^{*}(g)-v_{3}^{*}(g-23)-c \geq 0 \\
& \Leftrightarrow \quad \frac{n-1}{2 n}-\frac{n-3}{2(n-2)}-\frac{1}{2 n} \geq 0
\end{aligned}
$$

\footnotetext{
${ }^{29}$ Recall that some $i \in N$ is said to be a loose-end player if it is $\eta_{i}(g)=1$, that is if she has exactly one link in $g$.
} 


$$
\Leftrightarrow \quad \frac{4-n}{2 n(n-2)} \geq 0
$$

Obviously, this is never fulfilled for $n \geq 5$, meaning that a line network cannot be pairwise stable.

Subclass (b):

Let $k \in N$ be a player with at least three neighbors including two or more loose-end players. Then Manea (2011a, Theorem 3) implies that it is $v_{k}^{*}(g) \geq \frac{2}{3}$. So it must be $k \in L_{1}$. Select a player $i \in N_{k}(g)$ such that $\eta_{i}(g) \geq \eta_{i^{\prime}}(g)$ for all $i^{\prime} \in N_{k}(g)$. Note that in the network $g-k i$, player $k$ still has at least two loose-end contacts such that again according to Manea (2011a, Theorem 3$)$ we have $v_{k}^{*}(g-k i) \geq \frac{2}{3}$. The corresponding stability condition then gives

$$
u_{k}^{*}(g) \geq u_{k}^{*}(g-k i) \Leftrightarrow v_{k}^{*}(g)-c \geq v_{k}^{*}(g-k i) \Rightarrow 1-x_{1}-c \geq \frac{2}{3} \Leftrightarrow x_{1}+c \leq \frac{1}{3} .
$$

This obviously contradicts equation (A.1). Thus, a network $g$ belonging to Subclass (b) cannot be pairwise stable.

Subclass (c):

First deliberate the following: For any tree network $\tilde{g}$ and any player $k \in N$ there exists a unique partition $\left(B r_{\nu}^{k}\right)_{\nu \in N_{k}(\tilde{g})}$ of $N \backslash\{k\}$ such that for all $\nu \in N_{k}(\tilde{g})$ it is $\nu \in B r_{\nu}^{k}$ and $\left.\tilde{g}\right|_{B r_{\nu}^{k}}$ is connected, i.e. $\left.\tilde{g}\right|_{B r_{\nu}^{k}}$ has only one component (if one restricts the player set to $B r_{\nu}^{k}$ ). Based on this observation, we define the subnetworks $\left(\left.\tilde{g}\right|_{B r_{\nu}^{k}}\right)_{\nu \in N_{k}(g)}$ to be the branches of player $k$ in $\tilde{g}$ and $\nu \in N_{k}(\tilde{g})$ is said to be the fork player of $\left.\tilde{g}\right|_{B r_{\nu}^{k}}$.

Note that if $g$ belongs to Subclass (c), then there exists a player $k \in N$ who has more than two links such that for at least all but one of her branches, all players contained in these have at most two links in $g$. If this would not be the case, the following procedure would never stop, meaning that there would have to be infinitely many players in $N$ : Initially, select a player $k_{0}$ having more than two links and one of her branches containing another player $k_{1}$ with more than two links. Then by assumption player $k_{1}$ must have a branch in $g$ which does not contain player $k_{0}$ but a player $k_{2}$ who also has more than two links. For this player $k_{2}$ there must again be a branch in $g$ not containing $k_{0}$ and $k_{1}$ but a player $k_{3}$ having more than two links. Continuing this way, for any $m \in \mathbb{N}$ there is a player $k_{m+1} \in N \backslash\left\{k_{0}, \ldots, k_{m}\right\}$, which then gives a contradiction by induction. Thus, a player $k$ as mentioned above must indeed exist.

In the following we distinguish two cases.

Case (c).1: $k \in L_{1}$

If there are other players having more than two links, then let $i \in N$ be the fork player of player $k$ 's branch which contains all of them. Otherwise, arbitrarily pick some $i \in N_{k}(g)$. In both cases consider the network $g-k i$ and the component $C \subset N$ which player $k$ is 
contained in. In the network $\left.g\right|_{C}$, there is only player $k$ who might have more than two links. Furthermore, every branch of player $k$ in $\left.g\right|_{C}$ must be a line of odd length as Manea (2011a, Theorem 3) implies that any loose-end player in $g$ is contained in $M_{1}$. In turn, this implies that for any $\left.g\right|_{C}$-independent set $M$ with $\frac{\left|L^{g \mid C}(M)\right|}{|M|}<1$ it is $k \in L^{\left.g\right|_{C}}(M)$. One example for such a set is $M_{1} \cap C$ with partner set $L_{1} \cap C$. Hence, it must be $v_{k}^{*}(g-k i)>\frac{1}{2}$. The corresponding stability condition then gives

$$
u_{k}^{*}(g) \geq u_{k}^{*}(g-k i) \Leftrightarrow v_{k}^{*}(g)-c \geq v_{k}^{*}(g-k i) \Rightarrow 1-x_{1}-c>\frac{1}{2} \Leftrightarrow x_{1}+c<\frac{1}{2} .
$$

This obviously again contradicts equation (A.1). Consequently, a network $g$ belonging to Subclass (c) with $k \in L_{1}$ cannot be pairwise stable.

Case (c).2:

We need to introduce some additional notation here. Identify a branch of player $k$ which is a line network with minimal length among all of these line branches. We denote the set of players in this branch by $B^{1} \subset N$. Note that any branch of player $k$ which is a line must be of even length. Let $\hat{M}^{1}:=M_{1} \cap B^{1}$ and $\hat{L}^{1}:=L_{1} \cap B^{1}$. Then it is $\left|\hat{M}^{1}\right|=\left|\hat{L}^{1}\right|$. Let $j$ denote the fork player of this branch. In addition, let $B^{2} \subset N$ denote the set of all players contained in the other line branch(es) of player $k$. Let similarly $\hat{M}^{2}:=M_{1} \cap B^{2}$ and $\hat{L}^{2}:=L_{1} \cap B^{2}$. Then we have $\left|\hat{M}^{2}\right|=\left|\hat{L}^{2}\right| \geq\left|\hat{M}^{1}\right|$. Finally, let $B^{3}:=N \backslash\left(B^{1} \dot{\cup} B^{2} \dot{\cup}\{k\}\right)$ and $\hat{M}^{3}:=M_{1} \cap B^{3}$, $\hat{L}^{3}:=L_{1} \cap B^{3}$. Then it must be $\left|\hat{M}^{3}\right| \geq\left|\hat{L}^{3}\right|$ as we have $\left|M_{1}\right|>\left|L_{1}\right|$.

Note that we must have $r_{1}=\frac{\left|L_{1}\right|}{\left|M_{1}\right|} \leq \frac{\left|\hat{L}^{3}\right|}{\left|\hat{M}^{3}\right|}$ since $r_{1}$ is the minimal shortage ratio for $g$ and obviously $L^{g}\left(\hat{M}^{3}\right)=\hat{L}^{3}$. Thus, applying the above notation gives

$$
x_{1}=\frac{\left|L_{1}\right|}{\left|M_{1}\right|+\left|L_{1}\right|}=\frac{\left|\hat{M}^{1}\right|+\left|\hat{M}^{2}\right|+\left|\hat{L}^{3}\right|}{2\left|\hat{M}^{1}\right|+2\left|\hat{M}^{2}\right|+\left|\hat{M}^{3}\right|+\left|\hat{L}^{3}\right|+1} .
$$

Now consider the network $g^{\prime}:=g-k j$ and let $\left(r_{s}^{\prime}, x_{s}^{\prime}, M_{s}^{\prime}, L_{s}^{\prime}, N_{s}^{\prime}, g_{s}^{\prime}\right)_{s=1, \ldots, \bar{s}^{\prime}}$ be the outcome of the algorithm $\mathcal{A}\left(g^{\prime}\right)$. Notice first that the set $\hat{M}^{2} \dot{\cup} \hat{M}^{3} \dot{\cup}\{k\} \subset M_{1}$ is $g^{\prime}$-independent and $\hat{L}^{2} \dot{\cup} \hat{L}^{3}$ is the corresponding partner set in $g^{\prime}$. Furthermore, we have

$$
\frac{\left|\hat{L}^{2}\right|+\left|\hat{L}^{3}\right|}{\left|\hat{M}^{2}\right|+\left|\hat{M}^{3}\right|+1}=\frac{\left|\hat{M}^{2}\right|+\left|\hat{L}^{3}\right|}{\left|\hat{M}^{2}\right|+\left|\hat{M}^{3}\right|+1}<1 .
$$

Assume for contradiction that there is another $g^{\prime}$-independent set $M^{\prime} \subseteq N$ with partner set $L^{\prime}=L^{g^{\prime}}\left(M^{\prime}\right) \subseteq N$ which is shortage ratio minimizing in step $s=1$ of $\mathcal{A}\left(g^{\prime}\right)$. Since the set $B^{1}$ is a component of $g^{\prime}$ and it induces a line network of even length where every player receives a payoff of $\frac{1}{2}$, we must have $\left(M^{\prime} \cup L^{\prime}\right) \cap B^{1}=\emptyset$ and $\bar{s}^{\prime} \geq 2$. Moreover, Lemma 2 yields that $M_{1} \cap L_{s}^{\prime}=L_{1} \cap M_{s}^{\prime}=\emptyset$ for all $s<\bar{s}^{\prime}$. Hence, we must have $M^{\prime} \subset \hat{M}^{2} \dot{\cup} \hat{M}^{3} \dot{\cup}\{k\}$ 
and $L^{\prime} \subset \hat{L}^{2} \dot{\cup} \hat{L}^{3}$ such that

$$
\frac{\left|L^{\prime}\right|}{\left|M^{\prime}\right|}<\frac{\left|\hat{M}^{2}\right|+\left|\hat{L}^{3}\right|}{\left|\hat{M}^{2}\right|+\left|\hat{M}^{3}\right|+1}<1
$$

On the contrary, $M^{\prime} \dot{\cup} \hat{M}^{1} \subset M_{1}$ is $g$-independent and we have $L^{g}\left(M^{\prime} \dot{\cup} \hat{M}^{1}\right)=L^{\prime} \dot{\cup} \hat{L}^{1}$. The minimality of $r_{1}=\frac{\left|L_{1}\right|}{\left|M_{1}\right|}$ in $\mathcal{A}(g)$ then implies

$$
r_{1}=\frac{\left|\hat{M}^{2}\right|+\left|\hat{L}^{3}\right|+\left|\hat{M}^{1}\right|}{\left|\hat{M}^{2}\right|+\left|\hat{M}^{3}\right|+1+\left|\hat{M}^{1}\right|} \leq \frac{\left|L^{\prime}\right|+\left|\hat{M}^{1}\right|}{\left|M^{\prime}\right|+\left|\hat{M}^{1}\right|}<1 \quad \Rightarrow \quad \frac{\left|\hat{M}^{2}\right|+\left|\hat{L}^{3}\right|}{\left|\hat{M}^{2}\right|+\left|\hat{M}^{3}\right|+1} \leq \frac{\left|L^{\prime}\right|}{\left|M^{\prime}\right|} .
$$

Thus, arriving at a contradiction, this implies that

$$
v_{k}^{*}\left(g^{\prime}\right)=\frac{\left|\hat{M}^{2}\right|+\left|\hat{L}^{3}\right|}{2\left|\hat{M}^{2}\right|+\left|\hat{M}^{3}\right|+\left|\hat{L}^{3}\right|+1} .
$$

Taking into account equation (A.2), the corresponding stability condition demands

$$
\begin{aligned}
u_{k}^{*}(g) \geq u_{k}^{*}(g-k j) & \Leftrightarrow \quad v_{k}^{*}(g)-\eta_{k}(g) c \geq v_{k}^{*}\left(g^{\prime}\right)-\eta_{k}\left(g^{\prime}\right) c \\
& \Leftrightarrow \quad x_{1} \geq v_{k}^{*}\left(g^{\prime}\right)+\frac{1}{2}-x_{1} \\
& \Leftrightarrow \quad 2 x_{1}-v_{k}^{*}\left(g^{\prime}\right) \geq \frac{1}{2}
\end{aligned}
$$

However, we now establish that it must be $2 x_{1}-v_{k}^{*}\left(g^{\prime}\right)<\frac{1}{2}$. Recalling equations (A.3) and (A.4), some calculation yields

$$
\begin{aligned}
2 x_{1}-v_{k}^{*}\left(g^{\prime}\right) & =\frac{2\left|\hat{M}^{1}\right|+2\left(\left|\hat{M}^{2}\right|+\left|\hat{L}^{3}\right|\right)}{2\left|\hat{M}^{1}\right|+\left(2\left|\hat{M}^{2}\right|+\left|\hat{M}^{3}\right|+\left|\hat{L}^{3}\right|+1\right)}-\frac{\left(\left|\hat{M}^{2}\right|+\left|\hat{L}^{3}\right|\right)}{\left(2\left|\hat{M}^{2}\right|+\left|\hat{M}^{3}\right|+\left|\hat{L}^{3}\right|+1\right)} \\
& =\frac{2\left|\hat{M}^{1}\right|\left(\left|\hat{M}^{2}\right|+\left|\hat{M}^{3}\right|+1\right)+\left(\left|\hat{M}^{2}\right|+\left|\hat{L}^{3}\right|\right)\left(2\left|\hat{M}^{2}\right|+\left|\hat{M}^{3}\right|+\left|\hat{L}^{3}\right|+1\right)}{2\left|\hat{M}^{1}\right|\left(2\left|\hat{M}^{2}\right|+\left|\hat{M}^{3}\right|+\left|\hat{L}^{3}\right|+1\right)+\left(2\left|\hat{M}^{2}\right|+\left|\hat{M}^{3}\right|+\left|\hat{L}^{3}\right|+1\right)^{2}} \\
& =\frac{D-R}{2 D}
\end{aligned}
$$

where

$$
D=2\left|\hat{M}^{1}\right|\left(2\left|\hat{M}^{2}\right|+\left|\hat{M}^{3}\right|+\left|\hat{L}^{3}\right|+1\right)+\left(2\left|\hat{M}^{2}\right|+\left|\hat{M}^{3}\right|+\left|\hat{L}^{3}\right|+1\right)^{2}>0
$$

and

$$
\begin{aligned}
& R=-2\left|\hat{M}^{1}\right|\left|\hat{M}^{3}\right|+2\left|\hat{M}^{1}\right|\left|\hat{L}^{3}\right|-2\left|\hat{M}^{1}\right|+2\left|\hat{M}^{2}\right|\left|\hat{M}^{3}\right|-2\left|\hat{M}^{2}\right|\left|\hat{L}^{3}\right|+2\left|\hat{M}^{2}\right|+\left|\hat{M}^{3}\right|^{2} \\
&+2\left|\hat{M}^{3}\right|-\left|\hat{L}^{3}\right|^{2}+1 \\
&= 2(\underbrace{\left|\hat{M}^{2}\right|-\left|\hat{M}^{1}\right|}_{\geq 0})+2(\underbrace{\left|\hat{M}^{3}\right|-\left|\hat{L}^{3}\right|}_{\geq 0})(\underbrace{\left|\hat{M}^{2}\right|-\left|\hat{M}^{1}\right|}_{\geq 0})+(\underbrace{\left|\hat{M}^{3}\right|^{2}-\left|\hat{L}^{3}\right|^{2}}_{\geq 0})+2\left|\hat{M}^{3}\right|+1 \\
& \geq 2\left|\hat{M}^{3}\right|+1
\end{aligned}
$$


$>0$.

Hence, we indeed have

$$
2 x_{1}-v_{k}^{*}(g-k j)=\frac{D-R}{2 D}<\frac{1}{2} .
$$

This concludes the proof for Subclass (c) and of the whole proposition.

Proof of Proposition 2(ii). Consider a pairwise stable network $g$ and assume that there is a cut-player $k \in N$ who is part of a cycle and receives a payoff $v_{k}^{*}(g)>\frac{1}{2}$. According to Proposition 1 and Corollary 2, the algorithm $\mathcal{A}(g)$ must stop after the first step providing an outcome $\left(r_{1}, x_{1}, M_{1}, L_{1}, N_{1}, g_{1}\right)$ with $M_{1} \dot{\cup} L_{1}=N,\left|M_{1}\right|>\left|L_{1}\right|$ and $\left.g\right|_{M_{1}}=\left.g\right|_{L_{1}}=\emptyset$. So we have $r_{1}=\frac{\left|L_{1}\right|}{\left|M_{1}\right|} \in(0,1), k \in L_{1}$ and $v_{k}^{*}(g)=1-x_{1}$. Further, by Theorem 3 it is $x_{1}+c=\frac{1}{2}$.

In what follows, we prove that player $k$ can delete a certain link such that in the resulting network she still receives a payoff greater than $\frac{1}{2}$. To start with, note that by assumption there must be a set $K \subset N$ with $k \in K$ such that

- $L^{g}(K \backslash\{k\}) \subseteq K$,

- $k$ is contained in a cycle in $\left.g\right|_{K^{\complement} \cup\{k\}}$ and

- $\left.g\right|_{K^{\mathrm{C}}}$ has only one component (as usual, considering $K^{\complement}$ as player set).

As $g$ has only one component, it must be $k \in L^{g}(K \backslash\{k\})$, meaning that $N_{k}(g) \cap K \neq \emptyset$. Moreover, there exists $i^{\prime} \in N_{k}(g) \backslash K$ such that $k$ and $i^{\prime}$ belong to the same cycle in $g$. Now consider the network $g^{\prime}:=g-k i^{\prime}$ and let $\left(r_{s}^{\prime}, x_{s}^{\prime}, M_{s}^{\prime}, L_{s}^{\prime}, N_{s}^{\prime}, g_{s}^{\prime}\right)_{s=1, \ldots, \bar{s}^{\prime}}$ be the outcome of $\mathcal{A}\left(g^{\prime}\right)$. Lemma 2 yields that $v_{k}^{*}\left(g^{\prime}\right) \geq \frac{1}{2}$. Assume for contradiction that we have $v_{k}^{*}\left(g^{\prime}\right)=\frac{1}{2}$, meaning that $k \in N_{\bar{s}^{\prime}}^{\prime}$.

Consider the set $C_{k}^{\prime}:=C_{k}\left(\left.g_{\bar{s}^{\prime}}^{\prime}\right|_{K}\right)=C_{k}\left(\left.g\right|_{N_{\bar{s}^{\prime}}^{\prime} \cap K}\right)$, that is the component of player $k$ in the network $g$ restricted to the set $N_{\bar{s}^{\prime}}^{\prime} \cap K$. As a first step, we establish that it is

$$
\frac{\left|L_{1} \cap C_{k}^{\prime}\right|}{\left|M_{1} \cap C_{k}^{\prime}\right|}=1
$$

Note first that we have $N_{k}\left(\left.g^{\prime}\right|_{K}\right) \neq \emptyset$. Furthermore, it must be $N_{k}\left(g^{\prime}\right) \subseteq M_{1} \cap N_{\bar{s}^{\prime}}^{\prime}$ as Lemma 2 yields $M_{1} \cap L_{s}^{\prime}=\emptyset$ for all $s<\bar{s}^{\prime}$. This guarantees that $M_{1} \cap C_{k}^{\prime} \neq \emptyset$. Based on this, we can immediately rule out the possibility that the left-hand side of (A.6) is strictly smaller than one since $M_{1} \cap C_{k}^{\prime}$ is $g^{\prime}$-independent and clearly $L^{g_{s^{\prime}}^{\prime}}\left(M_{1} \cap C_{k}^{\prime}\right) \subseteq L_{1} \cap C_{k}^{\prime}$. So assume that the left-hand side of (A.6) is strictly greater than one. We make use of the following implication which we verify at the end of the proof:

$$
\begin{aligned}
&|\hat{L}|=|\hat{M}| \geq 1 \text { for } \hat{L} \subseteq L_{1} \cap C_{k}^{\prime} \backslash\{k\}, N_{k}(g) \cap K \subseteq \hat{M} \subseteq M_{1} \cap C_{k}^{\prime} \\
& \Rightarrow L^{g_{\bar{s}^{\prime}}^{\prime}(\hat{L}) \backslash \hat{M} \neq \emptyset}
\end{aligned}
$$


We know that it is $\emptyset \neq N_{k}(g) \cap K \subseteq N_{k}\left(g^{\prime}\right) \subseteq N_{\bar{s}^{\prime}}^{\prime}$. Let $\hat{M}^{0}:=N_{k}(g) \cap K$ such that $\hat{M}^{0} \subseteq M_{1} \cap C_{k}^{\prime}$. Hence, it must be $\left|L_{1} \cap C_{k}^{\prime} \backslash\{k\}\right| \geq\left|\hat{M}^{0}\right|$ since otherwise we would get

$$
\frac{\left|L_{1} \cap C_{k}^{\prime}\right|}{\left|M_{1} \cap C_{k}^{\prime}\right|} \leq \frac{\left|L_{1} \cap C_{k}^{\prime}\right|}{\left|\hat{M}^{0}\right|} \leq 1
$$

that is a contradiction to our assumption. So select a set of players $\hat{L}^{0} \subseteq L_{1} \cap C_{k}^{\prime} \backslash\{k\}$ with $\left|\hat{L}^{0}\right|=\left|\hat{M}^{0}\right|$. Note that $\hat{M}^{0}$ and $\hat{L}^{0}$ satisfy the conditions of implication (A.7).

Based on this, we can construct a sequence of players $\left(j_{1}, j_{2}, j_{3}, \ldots\right)$ in a certain way such that according to the previous considerations, the underlying procedure which sequentially adds players to the sequence can never break up. As in the proofs of Lemma 1 and 2, this leads to a contradiction to the finiteness of the player set $N$. Given such a sequence, let $\hat{M}^{m}:=\left\{j_{l} \mid 1 \leq l \leq m, l\right.$ odd $\} \cup \hat{M}^{0}$ and $\hat{L}^{m}:=\left\{j_{l} \mid 1 \leq l \leq m, l\right.$ even $\} \cup \hat{L}^{0}$ for $m \in \mathbb{N}$. Now consider some even number $m \in \mathbb{N} \cup\{0\}$. Assume that $\hat{L}^{m} \subseteq L_{1} \cap C_{k}^{\prime} \backslash\{k\}$, $N_{k}(g) \cap K \subseteq \hat{M}^{m} \subseteq M_{1} \cap C_{k}^{\prime}$ and $\left|\hat{L}^{m}\right|=\left|\hat{M}^{m}\right| \geq 1$. We then have:

- By implication (A.7) there exists $j_{m+1} \in L^{g_{\bar{s}^{\prime}}^{\prime}}\left(\hat{L}^{m}\right) \backslash \hat{M}^{m}$. For this player it must hold that $j_{m+1} \in M_{1} \cap C_{k}^{\prime} \backslash \hat{M}^{m}$ since $\hat{L}^{m} \subseteq L_{1} \cap C_{k}^{\prime} \backslash\{k\}$.

- Then there must exist $j_{m+2} \in L_{1} \cap C_{k}^{\prime} \backslash\left(\hat{L}^{m+1} \dot{\cup}\{k\}\right)$ since otherwise we would have

$$
1<\frac{\left|L_{1} \cap C_{k}^{\prime}\right|}{\left|M_{1} \cap C_{k}^{\prime}\right|} \leq \frac{\left|\hat{L}^{m+1} \dot{\cup}\{k\}\right|}{\left|\hat{M}^{m+1}\right|}=1 .
$$

Thus it is $\hat{L}^{m+2} \subseteq L_{1} \cap C_{k}^{\prime} \backslash\{k\}, N_{k}(g) \cap K \subseteq \hat{M}^{m+2} \subseteq M_{1} \cap C_{k}^{\prime}$ and $\left|\hat{L}^{m+2}\right|=\left|\hat{M}^{m+2}\right|=$ $\left|\hat{L}^{m}\right|+1 \geq 1$. By induction this leads to a contradiction to the finiteness of the player set $N$. This establishes equation (A.6), however, under the assumption of having $v_{k}^{*}\left(g^{\prime}\right)=\frac{1}{2}$.

During the second step we now use this to construct a concluding contradiction of similar kind arising from the assumption that $v_{k}^{*}\left(g^{\prime}\right)=\frac{1}{2}$. Here, we make use of the following implication:

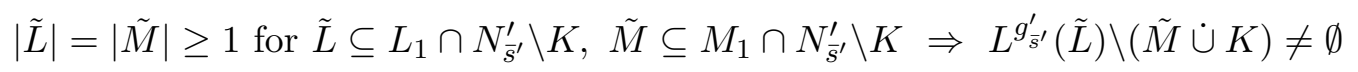

Its verification is postponed to the end of this proof as well. Note that by definition it is $\frac{\left|L^{g^{\prime}}(\bar{M})\right|}{|M|} \geq 1$ for all $g^{\prime}$-independent sets $\bar{M} \subseteq N_{\bar{s}^{\prime}}^{\prime}$. Based on this, we can again construct a sequence of players $\left(i_{1}, i_{2}, i_{3}, \ldots\right)$ such that, according to the previous considerations, the sequential adding of new players can never break up. Thus, we again get a contradiction to the finiteness of the player set $N$. For this purpose, we define the sets $\tilde{M}^{m}:=\left\{i_{l} \mid 1 \leq l \leq\right.$ $m, l$ odd $\}$ and $\tilde{L}^{m}:=\left\{i_{l} \mid 1 \leq l \leq m, l\right.$ even $\}$ for $m \in \mathbb{N}$.

Initially, select a player $i_{1} \in M_{1} \cap N_{\bar{s}^{\prime}}^{\prime} \backslash K$. Such a player must exist as $k \in L_{1} \cap N_{\bar{s}^{\prime}}^{\prime}$ is part of a cycle in $\left.g\right|_{N \backslash K \cup\{k\}}$ and, according to Lemma 2, we have $M_{1} \cap L_{s}^{\prime}=\emptyset$ for all $s<\bar{s}^{\prime}$. Now consider some odd number $m \in \mathbb{N}$. Assume that $\tilde{M}^{m} \subseteq M_{1} \cap N_{\bar{s}^{\prime}}^{\prime} \backslash K, \tilde{L}^{m} \subseteq L_{1} \cap N_{\bar{s}^{\prime}}^{\prime} \backslash K$ 
and that $\left|\tilde{M}^{m}\right|=\frac{m+1}{2}>\frac{m-1}{2}=\left|\tilde{L}^{m}\right|$. We then have:

- $\tilde{M}^{m} \dot{\cup}\left(M_{1} \cap C_{k}^{\prime}\right) \subseteq N_{\bar{s}^{\prime}}^{\prime}$ is $g^{\prime}$-independent and

$$
\frac{\left|\tilde{L}^{m} \dot{\cup}\left(L_{1} \cap C_{k}^{\prime}\right)\right|}{\left|\tilde{M}^{m} \dot{\cup}\left(M_{1} \cap C_{k}^{\prime}\right)\right|}<1
$$

since it is $\left|L_{1} \cap C_{k}^{\prime}\right|=\left|M_{1} \cap C_{k}^{\prime}\right|$ as we know from equation (A.6). As we have $k \in L^{g_{s^{\prime}}^{\prime}}\left(M_{1} \cap C_{k}^{\prime}\right) \subseteq L_{1} \cap C_{k}^{\prime}$, this implies that there must exist a player $i_{m+1} \in$ $L^{g_{\bar{s}^{\prime}}^{\prime}}\left(\tilde{M}^{m}\right) \backslash\left(\tilde{L}^{m} \dot{\cup} K\right)$. It is $i_{m+1} \in L_{1} \cap N_{\bar{s}^{\prime}}^{\prime} \backslash\left(\tilde{L}^{m} \dot{\cup} K\right)$ since $\tilde{M}^{m} \subseteq M_{1}$.

- We then have $\left|\tilde{L}^{m+1}\right|=\left|\tilde{M}^{m+1}\right|=\frac{m+1}{2} \geq 1$ and $\tilde{L}^{m+1} \subseteq L_{1} \cap N_{\bar{s}^{\prime}}^{\prime} \backslash K, \tilde{M}^{m+1} \subseteq$

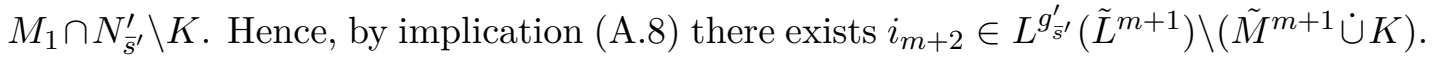
It is $i_{m+2} \in M_{1} \cap N_{\bar{s}^{\prime}}^{\prime} \backslash\left(\tilde{M}^{m+1} \dot{\cup} K\right)$ since $\tilde{L}^{m+1} \subseteq L_{1}$.

Thus, we have $\tilde{M}^{m+2} \subseteq M_{1} \cap N_{\bar{s}^{\prime}}^{\prime} \backslash K, \tilde{L}^{m+2} \subseteq L_{1} \cap N_{\bar{s}^{\prime}}^{\prime} \backslash K$ and $\left|\tilde{M}^{m+2}\right|=\frac{(m+2)+1}{2}>$ $\frac{(m+2)-1}{2}=\left|\tilde{L}^{m+2}\right|$. Again, by induction this leads to a contradiction to the finiteness of the player set $N$. This proves that player $k$ 's payoff must indeed be strictly greater than $\frac{1}{2}$. The corresponding stability condition then yields

$$
u_{k}^{*}(g) \geq u_{k}^{*}\left(g-k i^{\prime}\right) \Leftrightarrow v_{k}^{*}(g)-c \geq v_{k}^{*}\left(g^{\prime}\right) \Rightarrow 1-x_{1}-c>\frac{1}{2} \Leftrightarrow x_{1}+c<\frac{1}{2},
$$

which is a contradiction to Theorem 3. Hence, such a network $g$ cannot be pairwise stable.

It remains to prove implications (A.7) and (A.8). We start with the first one. Given the two sets $\hat{L} \subseteq L_{1} \cap C_{k}^{\prime} \backslash\{k\}$ and $\hat{M} \subseteq M_{1} \cap C_{k}^{\prime}$ with $N_{k}(g) \cap K \subseteq \hat{M}$ and $|\hat{L}|=|\hat{M}| \geq 1$ assume for contradiction that $L^{g_{\bar{s}^{\prime}}^{\prime}}(\hat{L}) \subseteq \hat{M}$. Note that we have $N_{j}\left(g_{\bar{s}^{\prime}}^{\prime}\right)=N_{j}(g)$ for all $j \in \hat{L}$ since it is $\hat{L} \subseteq L_{1} \cap N_{\bar{s}^{\prime}}^{\prime} \backslash\{k\}$ and, according to Lemma $2, M_{1} \cap L_{s}^{\prime}=\emptyset$ for all $s<\bar{s}^{\prime}$. Together with the assumption this implies that $L^{g}\left(M_{1} \cap K \backslash \hat{M}\right) \subseteq L_{1} \cap K \backslash \hat{L}$. Moreover, since $N_{k}(g) \cap K \subseteq \hat{M}$, it even is $L^{g}\left(M_{1} \cap K \backslash \hat{M}\right) \subseteq L_{1} \cap K \backslash(\hat{L} \dot{\cup}\{k\})$.

Additionally, we need the following inequalities:

$$
\frac{\left|L_{1} \cap K\right|-1}{\left|M_{1} \cap K\right|} \leq r_{1} \leq \frac{\left|L_{1} \cap K\right|}{\left|M_{1} \cap K\right|} \leq 1
$$

To see that these are correct, note first that it is $L^{g}\left(M_{1} \cap K\right) \subseteq L_{1} \cap K$ and similarly $L^{g}\left(M_{1} \backslash K\right) \subseteq L_{1} \backslash K \cup \dot{\cup}\{k\}$. So we must have $r_{1} \leq \frac{\left|L_{1} \cap K\right|}{\left|M_{1} \cap K\right|}$ and $r_{1} \leq \frac{\left|L_{1} \backslash K\right|+1}{\left|M_{1} \backslash K\right|}$ as $r_{1}$ is the minimal shortage ratio. Moreover, it is $r_{1}=\frac{\left|L_{1}\right|}{\left|M_{1}\right|}<1, M_{1}=\left(M_{1} \cap K\right) \dot{\cup}\left(M_{1} \backslash K\right)$ and $L_{1}=\left(L_{1} \cap K\right) \dot{\cup}\left(L_{1} \backslash K\right)$. Together this implies that $\frac{\left|L_{1} \cap K\right|-1}{\left|M_{1} \cap K\right|}=\frac{\left|L_{1}\right|-\left(\left|L_{1} \backslash K\right|+1\right)}{\left|M_{1}\right|-\left|M_{1} \backslash K\right|} \leq r_{1}$. In particular, this means that $\left|L_{1} \cap K\right|-1<\left|M_{1} \cap K\right|$ which in turn implies $\frac{\left|L_{1} \cap K\right|}{\left|M_{1} \cap K\right|} \leq 1$.

According to the third inequality in (A.9) we must have $M_{1} \cap K \backslash \hat{M} \neq \emptyset$ since otherwise it would be $\left|L_{1} \cap K\right| \leq\left|M_{1} \cap K\right|=|\hat{M}|=|\hat{L}|<|\hat{L} \dot{\cup}\{k\}| \leq\left|L_{1} \cap K\right|$. Taken together, this 
leads to the following contradiction:

$$
\begin{aligned}
r_{1} \leq \frac{\left|L^{g}\left(M_{1} \cap K \backslash \hat{M}\right)\right|}{\left|M_{1} \cap K \backslash \hat{M}\right|} & \leq \frac{\left|L_{1} \cap K \backslash(\hat{L} \dot{\cup}\{k\})\right|}{\left|M_{1} \cap K \backslash \hat{M}\right|} \\
& =\frac{\left|L_{1} \cap K\right|-|\hat{L}|-1}{\left|M_{1} \cap K\right|-|\hat{M}|} \\
& =\frac{\left|L_{1} \cap K\right|-1-|\hat{L}|}{\left|M_{1} \cap K\right|-|\hat{L}|}<\frac{\left|L_{1} \cap K\right|-1}{\left|M_{1} \cap K\right|} \leq r_{1},
\end{aligned}
$$

where the last two inequalities are due to (A.9) and the fact that $r_{1}<1$.

Similarly, to prove implication (A.8), we consider the two sets $\tilde{L} \subseteq L_{1} \cap N_{\bar{s}^{\prime}}^{\prime} \backslash K$ and $\tilde{M} \subseteq$ $M_{1} \cap N_{\bar{s}^{\prime}}^{\prime} \backslash K$ with $|\tilde{L}|=|\tilde{M}| \geq 1$ and assume for contradiction that we have $L^{g_{\bar{s}^{\prime}}^{\prime}}(\tilde{L}) \subseteq \tilde{M}$. Again according to Lemma 2, it must be $N_{j}\left(g_{\bar{s}^{\prime}}^{\prime}\right)=N_{j}(g)$ for all $j \in \tilde{L}$. Hence, we have that $L^{g}\left(M_{1} \backslash \tilde{M}\right) \subseteq L_{1} \backslash \tilde{L}$. Also, it is clear that $M_{1} \backslash \tilde{M} \neq \emptyset$ since otherwise we would have $\left|L_{1}\right|<\left|M_{1}\right|=|\tilde{M}|=|\tilde{L}| \leq\left|L_{1}\right|$. Summing up, this implies

$$
r_{1} \leq \frac{\left|L^{g}\left(M_{1} \backslash \tilde{M}\right)\right|}{\left|M_{1} \backslash \tilde{M}\right|} \leq \frac{\left|L_{1} \backslash \tilde{L}\right|}{\left|M_{1} \backslash \tilde{M}\right|}=\frac{\left|L_{1}\right|-|\tilde{L}|}{\left|M_{1}\right|-|\tilde{M}|}=\frac{\left|L_{1}\right|-|\tilde{L}|}{\left|M_{1}\right|-|\tilde{L}|}<\frac{\left|L_{1}\right|}{\left|M_{1}\right|}=r_{1},
$$

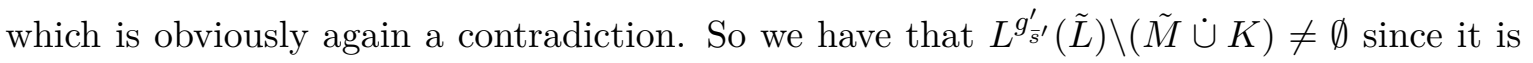
$L^{g_{\bar{s}^{\prime}}^{\prime}}(\tilde{L}) \subseteq K^{\complement}$. This concludes the proof.

Proof of Proposition 2(iii). Note first that, according to our characterization result, a component-induced subnetwork $g^{\prime}$ as mentioned in part (iii) could only be contained in a pairwise stable network if the algorithm $\mathcal{A}\left(g^{\prime}\right)$ stops after the first step. Let $\left(r_{1}, x, M_{1}, L_{1}, N_{1}, g_{1}\right)$ denote its outcome. By assumption, we obviously have $\left|M_{1}\right|=m$ and $\left|L_{1}\right|<\frac{m}{3}$. On the contrary, recalling stability condition (A.1), we get

$$
\frac{1}{4} \geq c=\frac{1}{2}-x=\frac{1}{2}-\frac{\left|L_{1}\right|}{\left|M_{1}\right|+\left|L_{1}\right|}=\frac{1}{2} \frac{\left|M_{1}\right|-\left|L_{1}\right|}{\left|M_{1}\right|+\left|L_{1}\right|} \Leftrightarrow 3\left|L_{1}\right| \geq\left|M_{1}\right| .
$$

Arriving at a contradiction, this proves the proposition's part (iii).

Proof of Proposition 2(iv). By Theorem 3, $g$ is connected and bipartite such that we have $v_{i}(g)=\frac{1}{2}-c$ for all $i \in M$ and $v_{j}(g)=\frac{1}{2}+c$ for all $j \in L$, implying that $\mathcal{A}(g)$ terminates after the first step. For some $i \in M$ and $j \in L$ consider now the network $g^{\prime}:=g-i j$ and let $\left(r_{s}^{\prime}, x_{s}^{\prime}, M_{s}^{\prime}, L_{s}^{\prime}, N_{s}^{\prime}, g_{s}^{\prime}\right)_{s^{\prime}=1, \ldots, \bar{s}^{\prime}}$ be the outcome of $\mathcal{A}\left(g^{\prime}\right)$. Note that $v_{i}^{*}\left(g^{\prime}\right)$ must be such that $0<c \leq v_{i}^{*}(g)-v_{i}^{*}\left(g^{\prime}\right)$, otherwise $i$ has an incentive to delete the link $i j$ contradicting pairwise stability of $g$. Furthermore, there exists an $\hat{s}^{\prime}<\bar{s}^{\prime}$ such that $i \in M_{\hat{s}^{\prime}}^{\prime}$ since $v_{i}\left(g^{\prime}\right)<v_{i}(g)<\frac{1}{2}$. Suppose $\hat{s}^{\prime} \neq 1$. For all $k \in M_{1}^{\prime}$ we then get $v_{k}^{*}\left(g^{\prime}\right)=\frac{\left|L_{1}^{\prime}\right|}{\left|M_{1}^{\prime}\right|+\left|L_{1}^{\prime}\right|}<\frac{\left|L_{\hat{s}}^{\prime}\right|}{\left|M_{\hat{s}^{\prime}}^{\prime}\right|+\left|L_{\hat{s}^{\prime}}^{\prime}\right|}=v_{i}^{*}\left(g^{\prime}\right)<$ $v_{i}^{*}(g)=v_{k}^{*}(g)$ (where the last equality holds because by Lemma 2 we have $k \in M$ ). The 
partner set of $M_{1}^{\prime}$ is the same in both networks $g$ and $g^{\prime}$ since $i \notin M_{1}^{\prime}$ and $M_{1}^{\prime} \subset M$ by Lemma 2. This is a contradiction to the minimality of the shortage ratio in the first step of the algorithm $\mathcal{A}(g)$ implying $i \in M_{1}^{\prime}$. Similarly, we have $j \notin L_{1}^{\prime}$ since otherwise by $v_{i}^{*}(g)<v_{i}^{*}\left(g^{\prime}\right)$ we get a contradiction to the minimality of the shortage ratio in the first step of $\mathcal{A}(g)$. Since $j \notin L_{1}^{\prime}$, we have $N_{j}(g) \cap M_{1}^{\prime} \backslash\{i\}=\emptyset$. The cardinalities $m^{\prime}:=\left|M_{1}^{\prime}\right|$ and $l^{\prime}:=\left|L_{1}^{\prime}\right|$ then determine the payoff of player $i$ in network $g^{\prime}$ such that $v_{i}^{*}\left(g^{\prime}\right)=\frac{l^{\prime}}{l^{\prime}+m^{\prime}}$. From $c \leq v_{i}^{*}(g)-v_{i}^{*}\left(g^{\prime}\right)$ and $v_{i}^{*}(g)=\frac{1}{2}-c$ we get $c \leq \frac{1}{2}\left(\frac{1}{2}-\frac{l^{\prime}}{l^{\prime}+m^{\prime}}\right)$ showing the first part.

For the second part, note that in network $g=g^{\prime}+i j$ we have that $M_{1}^{\prime}$ implies the partner set $L_{1}^{\prime} \cup\{j\}$. However, since the algorithm delivers $v_{i}^{*}(g)=\frac{|L|}{|M|+|L|}$ we must have $v_{i}^{*}(g) \leq \frac{\left|L_{1}^{\prime} \cup\{j\}\right|}{\left|M_{1}^{\prime}\right|+\left|L_{1}^{\prime} \cup\{j\}\right|}=\frac{l^{\prime}+1}{m^{\prime}+l^{\prime}+1}$. In particular, we then have

$$
\frac{l^{\prime}+1}{m^{\prime}+l^{\prime}+1}-\frac{l^{\prime}}{l^{\prime}+m^{\prime}} \geq v_{i}^{*}(g)-v_{i}^{*}\left(g^{\prime}\right) \geq c \quad \Leftrightarrow \quad \frac{m^{\prime}}{\left(m^{\prime}+l^{\prime}\right)^{2}+\left(m^{\prime}+l^{\prime}\right)} \geq c
$$

implying the second part.

\section{Acknowledgements}

The authors would like to thank Michael Günther, Christoph Kuzmics, Jakob Landwehr, Mihai Manea, Fernando Vega-Redondo, and anonymous reviewers, as well as the participants of various seminars at Bielefeld University and University Paris 1 Panthéon-Sorbonne for valuable comments and suggestions. This work was carried out within the International Research Training Group "Economic Behavior and Interaction Models" (EBIM) and was thus supported by the German Research Foundation (DFG) [contract GRK 1134/2].

\section{References}

Abreu, D., Manea, M., 2012a. Bargaining and efficiency in networks. Journal of Economic Theory 147 (1), 43-70.

Abreu, D., Manea, M., 2012b. Markov equilibria in a model of bargaining in networks. Games and Economic Behavior 75 (1), 1-16.

Baetz, O., 2015. Social activity and network formation. Theoretical Economics 10 (2), 315340.

Bala, V., Goyal, S., 2000. A noncooperative model of network formation. Econometrica 68 (5), 1181-1229.

Berge, C., 1981. Some common properties for regularizable graphs, edge-critical graphs and b-graphs. In: Saito, N., Nishizeki, T. (Eds.), Graph Theory and Algorithms. Springer, Berlin Heidelberg, pp. 108-123. 
Calvó-Armengol, A., 2003. Stable and efficient bargaining networks. Review of Economic Design 7 (4), 411-428.

Calvó-Armengol, A., 2004. Job contact networks. Journal of Economic Theory 115 (1), 191206.

Condorelli, D., Galeotti, A., 2012. Endogenous trading networks. University of Essex Department of Economics Discussion Paper No. 705.

Condorelli, D., Galeotti, A., forthcoming. Bilateral trading in networks. Review of Economic Studies.

Corominas-Bosch, M., 2004. Bargaining in a network of buyers and sellers. Journal of Economic Theory 115 (1), 35-77.

Elliott, M., 2015. Inefficiencies in networked markets. American Economic Journal: Microeconomics 7 (4), 43-82.

Gale, D., 1987. Limit theorems for markets with sequential bargaining. Journal of Economic Theory 43 (1), 20-54.

Galeotti, A., Goyal, S., Kamphorst, J., 2006. Network formation with heterogeneous players. Games and Economic Behavior 54 (2), 353-372.

Gauer, F., 2016. Strategic interaction and socio-economic structure. Ph.D. thesis, Bielefeld University.

Goyal, S., Joshi, S., 2003. Networks of collaboration in oligopoly. Games and Economic Behavior 43 (1), 57-85.

Goyal, S., Joshi, S., 2006. Bilateralism and free trade. International Economic Review 47 (3), 749-778.

Hellmann, T., 2013. On the existence and uniqueness of pairwise stable networks. International Journal of Game Theory 42 (1), 211-237.

Hellmann, T., Landwehr, J., 2014. Stable networks in homogeneous societies, Working Paper, Available at SSRN 2477452.

Jackson, M. O., Wolinsky, A., 1996. A strategic model of social and economic networks. Journal of Economic Theory 71 (1), 44-74.

Kranton, R., Minehart, D. F., 2001. A theory of buyer-seller networks. American Economic Review 91 (3), 485-508.

Manea, M., 2011a. Bargaining in stationary networks. American Economic Review 101 (5), 2042-2080. 
Manea, M., 2011b. Bargaining in stationary networks: Online appendix.

URL https://core.ac.uk/download/pdf/6309305.pdf

Manea, M., 2016. Models of bilateral trade in networks. In: The Oxford Handbook of the Economics of Networks. Citeseer.

Manea, M., forthcoming. Intermediation and resale in networks. Journal of Political Economy.

Nash, J. F., 1950. The bargaining problem. Econometrica 18 (2), 155-162.

Nash, J. F., 1953. Two-person cooperative games. Econometrica 21 (1), 128-140.

Polanski, A., 2007. Bilateral bargaining in networks. Journal of Economic Theory 134 (1), $557-565$.

Polanski, A., Vega-Redondo, F., 2013. Markets, bargaining, and networks with heterogeneous agents, Working Paper, Available at SSRN 2218605.

Rubinstein, A., 1982. Perfect equilibrium in a bargaining model. Econometrica 50 (1), 97109.

Rubinstein, A., Wolinsky, A., 1985. Equilibrium in a market with sequential bargaining. Econometrica 53 (5), 1133-1150.

Watts, A., 2001. A dynamic model of network formation. Games and Economic Behavior $34(2), 331-341$.

West, D. B., 2001. Introduction to Graph Theory. Vol. 2. Prentice-Hall, Englewood Cliffs, NJ. 